\title{
Optical photometry of the UCM Lists I and II
}

\section{The data}

\author{
P.G. Pérez-González, J. Zamorano, J. Gallego, and A. Gil de Paz
}

Departamento de Astrofísica, Universidad Complutense de Madrid, Av. Complutense s/n. 28040 Madrid, Spain e-mail: pag@astrax.fis.ucm.es, jaz@astrax.fis.ucm.es, jgm@astrax.fis.ucm.es, gil@astrax.fis.ucm.es

Received August 12; accepted November 8, 1999

\begin{abstract}
We present Johnson B CCD photometry for the whole sample of galaxies of the Universidad Complutense de Madrid (UCM) Survey Lists I and II. They constitute a well-defined and complete sample of galaxies in the Local Universe with active star formation. The data refer to 191 S0 to Irr galaxies at an averaged redshift of 0.027 , and complement the already published Gunn $r, J$ and $K$ photometries. In this paper the observational and reduction features are discussed in detail, and the new colour information is combined to search for clues on the properties of the galaxies, mainly by comparing our sample with other surveys.
\end{abstract}

Key words: galaxies: photometry — galaxies: fundamental parameters - surveys-galaxies-spiral, galaxies-structure - methods: data analysis

\section{Introduction}

The Universidad Complutense de Madrid Survey (UCM Survey List I; Zamorano et al. 1994, List II; Zamorano et al. 1996) constitutes a representative and fairly complete sample of current star-forming galaxies in the Local Universe (Gallego 1999). Its main purposes are to identify and study new young, low metallicity galaxies and to quantify the properties of the current star formation in the Local Universe. Another key goal was also to provide a reference sample for the studies of high-redshift populations, mainly dominated by star-forming galaxies.

The UCM Survey was carried out with the $80 / 120 \mathrm{~cm}$ $\mathrm{f} / 3$ Schmidt telescope at the German-Spanish Observatory of Calar Alto (Almería, Spain). A $4^{\circ}$ full-aperture prism

Send offprint requests to: P.G. Pérez-González

* Tables 1 and 3 are also available in electronic form at the CDS via anonymous ftp to cdsarc.u-strasbg.fr (130.79.128.5) or via http://cdsweb.u-strasbg.fr/Abstract.html and a IIIaF photographic emulsion were the standard instrumental setup. The survey was able to detect emission line galaxies (ELG) to a Gunn $r$ magnitude limit of about $18^{\mathrm{m}}$; the objects were selected by the presence of $\mathrm{H} \alpha \lambda 6563$ $+[\mathrm{NII}] \lambda 6584$ emission in their spectra. A total number of 191 objects were catalogued as UCM galaxies in List I and List II. A third list (UCM Survey List III; Alonso et al. 1999) will extend the sample around to $0.5^{\mathrm{m}}$ fainter objects due to the implementation of a new fully automatic procedure for the detection and analysis of the objectiveprism spectra.

The galaxies included in UCM lists I and II (hereafter the UCM survey) have been deeply analyzed in the Gunn $r$ bandpass (Vitores et al. 1996a and 1996b), and also in the $J$ and $K$ nIR bands by Alonso-Herrero et al. (1996) and Gil de Paz et al. (1999). The spectroscopic analysis was performed by (Gallego et al. 1996, 1997). It has also been used to deduce the $\mathrm{H} \alpha$ luminosity function in the Local Universe (Gallego et al. 1995). The UCM sample is now widely used as reference for spectroscopic studies of high- $z$ populations (see the nice review by Madau 1999).

The UCM survey includes a total of 191 galaxies at an averaged redshift of 0.027 . Morphologically, the sample is dominated by late-type spirals (around $47 \%$ being Sb or later) with less than $10 \%$ presenting typical parameters of earlier types and the remaining $10 \%$ being irregulars (Vitores et al. 1996a). Spectroscopically, all types of star-forming galaxies previously known in the literature are represented; most of the UCM objects are lowexcitation, high-metallicity starburst-like galaxies (57\%) but there are also high-excitation, low-metallicity HII-like galaxies (32\%). A fraction of AGN objects are also present $(8 \%)$. Their metallicities range from solar values to $\frac{1}{40} Z_{\odot}$, peaking at $\frac{1}{4} Z_{\odot}$ (Gallego et al. 1997).

Photometrically, the UCM Survey was first imaged in the Gunn $r$ band due to the close relationship between this band and the one used in the primary photographic plates. In order to get colour information of this sample, 
we started a long-term project to obtain detailed $B$ band photometry. This band was selected with two main purposes: (1) obtaining an optical colour with a considerable base width, (2) getting information more directly comparable with high-redshift surveys.

In this paper we present $B$ band photometry for the whole sample and compare it with the previous optical data. In later papers we will perform the study of the disk and bulge components in the $B$-band and will combine the broad band data (both optical and nIR) with $\mathrm{H} \alpha$ images in order to carry out a spatially resolved stellar population synthesis.

The paper is structured as follows: we introduce the sample of galaxies and the Johnson $B$ observations in Sect. 2. The galaxy photometry is afforded in Sect. 3 . Statistics and the comparison with previous photometry are considered in Sect. 4. Finally, we present the conclusions in Sect. 5. A Hubble constant $H_{0}=50 \mathrm{~km} \mathrm{~s}^{-1} \mathrm{Mpc}^{-1}$ and a deceleration parameter $q_{0}=0.5$ have been used throughout this paper.

\section{Observations}

\subsection{The sample}

The UCM sample of galaxies is divided into two lists. Galaxies of List I (Zamorano et al. 1994) and List II (Zamorano et al. 1996) were found in fields in a region of the sky from right ascension $12^{\mathrm{h}}$ to $16^{\mathrm{h}}$ and from $22^{\mathrm{h}}$ to $2^{\mathrm{h}}$, respectively. The surveyed region covered a $10^{\circ}$ width strip centered at declination $20^{\circ}$ for both lists. A summary of the main features of each galaxy is listed in Table 1, including the names, redshifts, morphological and spectral types, Gunn $r$ magnitudes and $B-V$ colour excesses as obtained from the Balmer decrements.

\subsection{The observations}

The whole sample was observed in six observing runs performed with three different telescopes. They were the $1.0 \mathrm{~m}$ Jacobus Kapteyn Telescope (JKT) at the Observatorio del Roque de los Muchachos in La Palma (Canary Islands, Spain), the $1.23 \mathrm{~m}$ telescope at the German-Spanish Observatory in Calar Alto (Almería, Spain) and the 1.52 meters Spanish Telescope in Calar Alto.

At the JKT we used a $1024 \times 1024$ CCD with a scale of $0.3^{\prime \prime} /$ pixel. The $1.52 \mathrm{~m}$ telescope in Calar Alto was equipped with a $1024 \times 1024 \mathrm{CCD}$ camera with a pixel size of 0 .' 4 . Finally, the 1.23 meters telescope images were taken with a $1024 \times 1024$ CCD camera with a scale of $0.5^{\prime \prime} /$ pixel and also with a $2048 \times 2048 \mathrm{CCD}$ with a pixel size of 0.313 .

Typical exposure times in the first three campaigns were $600 \mathrm{~s}$. Using this exposure time, the $24 \mathrm{mag}_{\operatorname{arcsec}}{ }^{-2}$ level was reached at $1 \sigma$ of the sky brightness. We increased exposure times to $1800 \mathrm{~s}$ in order to obtain deeper images. In this case, $2 \sigma$ of the sky brightness corresponded to $25 \mathrm{mag} \operatorname{arcsec}^{-2}$. Typical uncertainty (taking into account all sources of error) in the $B$ magnitude was always lower than $0.1 \mathrm{mag}$ in all campaigns.

All the objects were observed during photometric nights (most of them also dark) with seeing conditions ranging from $1^{\prime \prime} .0-1^{\prime \prime} .5$.

The main information of each observation campaign as well as the transformation equations that we will explain later are listed in Table 2.

\section{Galaxy photometry}

\subsection{Data reduction}

Standard reduction procedures for CCD photometry were applied. Once raw images were bias subtracted and flatfield corrected, cosmic rays were removed. The dark current was found to be negligible for all the cameras. During each night at least 10 bias images were obtained; in all cases they were very stable, so for each run we combined all of them to get an averaged bias that we subtracted to each image. We also took at least eight dome-flats that we combined and corrected from illumination failure with a combined sky-flat of at least six images. Finally cosmic rays were removed using the CR_UTILS IRAF ${ }^{1}$ package that replaced the values of the affected pixels by an interpolation of the surrounding pixels in an annulus. Foreground stars near the objects were also masked using a similar procedure.

\subsection{Flux calibration}

Integrated photometry was performed using the APPHOT IRAF package, mainly the polyphot and phot tasks. Standard Landolt (1992) stars observed during each night under different airmasses were used for calibration. They were measured with different apertures using the phot task. The curve of growth of each star was built following the algorithm found in Stetson (1990). A least-square method was used to get the following transformation equations:

$B-2.5 \cdot \log \left(F_{B}\right)=C+K_{B} \cdot X+K_{B-r} \cdot(B-r)$

where $B$ is the Johnson $B$ apparent magnitude, $F_{B}$ is the flux in counts s ${ }^{-1}, C$ is the instrumental constant, $K_{B}$ the extinction, $X$ the airmass, and $K_{B-r}$ the colour constant referred to the Johnson $B$-Gunn $r$ colour (we already had Gunn $r$ magnitudes of the galaxies).

\footnotetext{
${ }^{1}$ IRAF is distributed by the National Optical Astronomy Observatories, which are operated by the Association of Universities for Research in Astronomy, Inc. (AURA) under cooperative agreement with the National Science Foundation.
} 
Table 1. The sample of galaxies in the UCM survey Lists I and II

\begin{tabular}{|c|c|c|c|c|c|c|c|c|c|c|c|}
\hline $\begin{array}{l}\text { UCM name } \\
\text { (1) }\end{array}$ & $\begin{array}{c}z \\
(2)\end{array}$ & $\begin{array}{c}\text { MphT } \\
(3)\end{array}$ & $\begin{array}{l}\text { SpT } \\
(4)\end{array}$ & $\begin{array}{l}m_{\mathrm{r}} \\
(5)\end{array}$ & $\begin{array}{c}E(B-V) \\
(6)\end{array}$ & $\begin{array}{l}\text { UCM name } \\
\text { (1) }\end{array}$ & $\begin{array}{c}z \\
(2)\end{array}$ & $\begin{array}{c}\text { MphT } \\
(3)\end{array}$ & $\begin{array}{l}\text { SpT } \\
(4)\end{array}$ & $\begin{array}{l}m_{\mathrm{r}} \\
(5)\end{array}$ & $\begin{array}{c}E(B-V) \\
(6)\end{array}$ \\
\hline $0000+2140$ & 0.0238 & - & $\overline{\mathrm{HIIH}}$ & - & 1.204 & $1255+2734$ & 0.0234 & $\overline{\mathrm{Irr}}$ & $\overline{\mathrm{SBN}}$ & 15.99 & 0.715 \\
\hline $0003+2200$ & 0.0224 & $\mathrm{Sc}+$ & DANS & 16.16 & 0.867 & $1256+2717$ & 0.0273 & - & DHIIH & - & 0.447 \\
\hline $0003+2215$ & 0.0223 & - & $\mathrm{SBN}$ & - & 1.008 & $1256+2732$ & 0.0234 & S0 & $\mathrm{SBN}$ & 15.40 & - \\
\hline $0003+1955$ & 0.0278 & - & Sy1 & - & - & $1256+2701$ & 0.0247 & $\operatorname{Irr}$ & $\mathrm{HIIH}$ & 16.32 & 0.220 \\
\hline $0005+1802$ & 0.0187 & - & SBN & - & 1.244 & $1256+2910$ & 0.0279 & $\mathrm{Sb}$ & $\mathrm{SBN}$ & 15.10 & - \\
\hline $0006+2332$ & 0.0159 & - & $\mathrm{HIIH}$ & - & 0.644 & $1256+2823$ & 0.0307 & $\mathrm{Sb}$ & SBN & 15.11 & 0.644 \\
\hline $0013+1942$ & 0.0272 & $\mathrm{Sc}+$ & HIIH & 16.39 & 0.276 & $1256+2754$ & 0.0172 & - & SBN & - & 0.645 \\
\hline $0014+1829$ & 0.0182 & $\mathrm{Sa}$ & $\mathrm{HIIH}$ & 16.01 & 1.473 & $1256+2722$ & 0.0287 & $\mathrm{Sc}+$ & DANS & 16.05 & 0.928 \\
\hline $0014+1748$ & 0.0182 & $\mathrm{SBb}$ & $\mathrm{SBN}$ & 14.13 & 0.806 & $1257+2808$ & 0.0181 & $\mathrm{Sa}$ & $\mathrm{SBN}$ & 15.45 & 1.344 \\
\hline $0015+2212$ & 0.0198 & $\mathrm{Sa}$ & $\mathrm{HIIH}$ & 15.59 & 0.215 & $1258+2754$ & 0.0253 & $\mathrm{Sb}$ & $\mathrm{SBN}$ & 15.38 & 1.020 \\
\hline $0017+1942$ & 0.0281 & $\mathrm{Sc}+$ & HIIH & 15.34 & 0.357 & $1259+2934$ & 0.0239 & $\mathrm{Sb}$ & Sy2 & 14.18 & 0.984 \\
\hline $0017+2148$ & 0.0189 & - & $\mathrm{HIIH}$ & - & 0.575 & $1259+3011$ & 0.0307 & $\mathrm{Sa}$ & SBN & 15.36 & 0.682 \\
\hline $0018+2216$ & 0.0169 & $\mathrm{Sb}$ & DANS & 15.82 & 0.136 & $1259+2755$ & 0.0235 & $\mathrm{Sa}$ & $\mathrm{SBN}$ & 14.45 & 0.913 \\
\hline $0018+2218$ & 0.0220 & - & $\mathrm{SBN}$ & - & - & $1300+2907$ & 0.0219 & $\mathrm{Sb}$ & HIIH & 16.69 & 0.620 \\
\hline $0019+2201$ & 0.0191 & $\mathrm{Sc}+$ & DANS & 15.54 & 0.438 & $1301+2904$ & 0.0266 & $\mathrm{Sb}$ & $\mathrm{HIIH}$ & 15.18 & 0.207 \\
\hline $0022+2049$ & 0.0185 & $\mathrm{Sb}$ & HIIH & 14.45 & 0.901 & $1302+2853$ & 0.0237 & $\mathrm{Sa}$ & DHIIH & 15.77 & 0.621 \\
\hline $0023+1908$ & 0.0251 & - & HIIH & - & 0.409 & $1302+3032$ & 0.0342 & - & $\mathrm{HIIH}$ & - & 0.595 \\
\hline $0034+2119$ & 0.0315 & - & SBN & - & 0.684 & $1303+2908$ & 0.0261 & Irr & $\mathrm{HIIH}$ & 16.26 & - \\
\hline $0037+2226$ & 0.0204 & - & SBN & - & 0.615 & $1304+2808$ & 0.0210 & $\mathrm{Sa}$ & $\mathrm{SBN}$ & 14.85 & 0.114 \\
\hline $0038+2259$ & 0.0464 & $\mathrm{Sa}$ & SBN & 15.07 & 0.810 & $1304+2830$ & 0.0217 & $\mathrm{BCD}$ & DHIIH & 17.72 & 0.372 \\
\hline $0039+0054$ & 0.0191 & - & $\mathrm{SBN}$ & - & - & $1304+2907$ & 0.0159 & Irr & - & 14.55 & - \\
\hline $0040+0257$ & 0.0367 & $\mathrm{Sc}+$ & DANS & 16.76 & - & $1304+2818$ & 0.0244 & $\mathrm{Sc}+$ & SBN & 14.88 & 0.111 \\
\hline $0040+2312$ & 0.0254 & - & SBN & - & - & $1306+2938$ & 0.0211 & $\mathrm{Sb}$ & SBN & 14.80 & 0.501 \\
\hline $0040+0220$ & 0.0173 & $\mathrm{Sb}$ & DANS & 16.39 & 0.378 & $1306+3111$ & 0.0168 & $\mathrm{Sc}+$ & DANS & 15.32 & - \\
\hline $0040-0023$ & 0.0142 & LINER & - & - & - & $1307+2910$ & 0.0183 & $\mathrm{SBb}$ & $\mathrm{SBN}$ & 13.05 & 0.970 \\
\hline $0041+0134$ & 0.0169 & - & - & - & - & $1308+2958$ & 0.0223 & $\mathrm{Sc}+$ & SBN & 14.46 & 1.313 \\
\hline $0043+0245$ & 0.0180 & - & $\mathrm{HIIH}$ & - & 0.950 & $1308+2950$ & 0.0246 & $\mathrm{SBb}$ & SBN & 13.92 & 1.381 \\
\hline $0043-0159$ & 0.0161 & - & SBN & - & - & $1310+3027$ & 0.0234 & $\mathrm{Sa}$ & DANS & 15.70 & - \\
\hline $0044+2246$ & 0.0253 & $\mathrm{Sb}$ & $\mathrm{SBN}$ & 14.83 & 1.384 & $1312+3040$ & 0.0210 & $\mathrm{SBa}$ & SBN & 14.67 & 0.474 \\
\hline $0045+2206$ & 0.0203 & - & HIIH & - & 0.493 & $1312+2954$ & 0.0230 & $\mathrm{Sc}+$ & SBN & 15.14 & 1.087 \\
\hline $0047+2051$ & 0.0577 & $\mathrm{Sc}+$ & $\mathrm{SBN}$ & 16.00 & 0.598 & $1313+2938$ & 0.0380 & $\mathrm{Sa}$ & $\mathrm{HIIH}$ & 16.14 & - \\
\hline $0047-0213$ & 0.0144 & $\mathrm{Sa}$ & DHIIH & 14.82 & 0.857 & $1314+2827$ & 0.0253 & $\mathrm{Sa}$ & SBN & 15.54 & 0.749 \\
\hline $0047+2413$ & 0.0347 & $\mathrm{Sa}$ & $\mathrm{SBN}$ & 14.69 & 1.059 & $1320+2727$ & 0.0247 & $\mathrm{Sb}$ & DHIIH & 16.79 & 0.205 \\
\hline $0047+2414$ & 0.0347 & - & $\mathrm{SBN}$ & - & 0.592 & $1324+2926$ & 0.0172 & $\mathrm{BCD}$ & $\mathrm{BCD}$ & 16.85 & 0.022 \\
\hline $0049-0006$ & 0.0377 & $\mathrm{BCD}$ & $\mathrm{BCD}$ & 18.22 & 0.006 & $1324+2651$ & 0.0249 & S0 & $\mathrm{SBN}$ & 14.27 & 0.628 \\
\hline $0049+0017$ & 0.0140 & $\mathrm{Sc}+$ & DHIIH & 16.48 & 0.088 & $1331+2900$ & 0.0356 & $\mathrm{BCD}$ & $\mathrm{BCD}$ & 18.49 & 0.013 \\
\hline $0049-0045$ & 0.0048 & - & HIIH & - & 0.416 & $1428+2727$ & 0.0149 & $\mathrm{Sc}+$ & $\mathrm{HIIH}$ & 14.38 & 0.150 \\
\hline $0050+0005$ & 0.0346 & $\mathrm{Sa}$ & $\mathrm{HIIH}$ & 15.72 & 0.438 & $1429+2645$ & 0.0328 & $\mathrm{Sc}+$ & DHIIH & 16.91 & 0.105 \\
\hline $0050+2114$ & 0.0245 & $\mathrm{Sa}$ & $\mathrm{SBN}$ & 14.66 & 0.813 & $1430+2947$ & 0.0290 & So & HIIH & 15.95 & 0.308 \\
\hline $0051+2430$ & 0.0173 & - & SBN & - & 1.040 & $1431+2854$ & 0.0310 & $\mathrm{Sb}$ & SBN & 14.83 & - \\
\hline $0054-0133$ & 0.0512 & - & SBN & - & - & $1431+2702$ & 0.0384 & $\mathrm{Sb}$ & HIIH & 16.41 & 0.271 \\
\hline $0054+2337$ & 0.0164 & - & HIIH & - & 0.667 & $1431+2947$ & 0.0219 & $\mathrm{BCD}$ & BCD & 17.40 & - \\
\hline $0056+0044$ & 0.0183 & $\operatorname{Irr}$ & DHIIH & 16.58 & 0.079 & $1431+2814$ & 0.0320 & $\mathrm{Sa}$ & DANS & 15.85 & - \\
\hline $0056+0043$ & 0.0189 & $\mathrm{Sc}+$ & DHIIH & 16.07 & 0.331 & $1432+2645$ & 0.0307 & $\mathrm{SBb}$ & SBN & 14.59 & 0.914 \\
\hline $0119+2156$ & 0.0583 & $\mathrm{Sc}+$ & Sy2 & 15.44 & - & $1440+2521 \mathrm{~S}$ & 0.0314 & $\mathrm{Sb}$ & $\mathrm{SBN}$ & 16.16 & 0.292 \\
\hline $0121+2137$ & 0.0345 & $\mathrm{Sc}+$ & SBN & 15.41 & 0.703 & $1440+2511$ & 0.0333 & $\mathrm{Sb}$ & SBN & 15.87 & 1.018 \\
\hline $0129+2109$ & 0.0344 & - & LINER & - & - & $1440+2521 \mathrm{~N}$ & 0.0315 & $\mathrm{Sa}$ & SBN & 15.74 & 0.773 \\
\hline $0134+2257$ & 0.0353 & - & $\mathrm{SBN}$ & - & 0.892 & $1442+2845$ & 0.0110 & $\mathrm{Sb}$ & SBN & 14.66 & 0.681 \\
\hline $0135+2242$ & 0.0363 & So & DANS & 16.05 & 0.976 & $1443+2714$ & 0.0290 & $\mathrm{Sa}$ & Sy2 & 14.75 & 1.008 \\
\hline $0138+2216$ & 0.0591 & - & - & - & - & $1443+2844$ & 0.0279 & $\mathrm{SBc}$ & $\mathrm{SBN}$ & 14.91 & - \\
\hline $0141+2220$ & 0.0174 & $\mathrm{Sb}$ & DANS & 15.67 & 0.742 & $1443+2548$ & 0.0351 & $\mathrm{Sc}+$ & SBN & 15.12 & 0.726 \\
\hline $0142+2137$ & 0.0362 & $\mathrm{SBb}$ & Sy2 & 14.19 & 0.537 & $1444+2923$ & 0.0281 & SO & DANS & 15.77 & 0.785 \\
\hline $0144+2519$ & 0.0414 & $\mathrm{SB}(\mathrm{r})$ & SBN & 14.78 & 1.033 & $1452+2754$ & 0.0339 & $\mathrm{Sb}$ & SBN & 15.43 & 0.733 \\
\hline $0147+2309$ & 0.0194 & $\mathrm{Sa}$ & HIIH & 15.82 & 0.486 & $1506+1922$ & 0.0205 & $\mathrm{Sb}$ & $\mathrm{HIIH}$ & 14.87 & 0.453 \\
\hline $0148+2124$ & 0.0169 & $\mathrm{BCD}$ & $\mathrm{BCD}$ & 16.32 & 0.174 & $1513+2012$ & 0.0369 & So & $\mathrm{SBN}$ & 14.96 & 0.540 \\
\hline $0150+2032$ & 0.0323 & $\mathrm{Sc}+$ & HIIH & 16.28 & 0.085 & $1537+2506 \mathrm{~N}$ & 0.0231 & $\mathrm{SBb}$ & $\mathrm{HIIH}$ & 14.36 & 0.225 \\
\hline $0156+2410$ & 0.0134 & $\mathrm{Sc}+$ & DANS & 14.55 & 0.702 & $1537+2506 \mathrm{~S}$ & 0.0231 & $\mathrm{SBa}$ & HIIH & 15.50 & 0.357 \\
\hline $0157+2413$ & 0.0177 & $\mathrm{Sc}+$ & Sy2 & 13.65 & 0.725 & $1557+1423$ & 0.0275 & $\mathrm{Sb}$ & $\mathrm{SBN}$ & 15.82 & 0.374 \\
\hline $0157+2102$ & 0.0106 & $\mathrm{Sb}$ & HIIH & 14.39 & 0.474 & $1612+1308$ & 0.0114 & $\mathrm{BCD}$ & $\mathrm{BCD}$ & 17.48 & 0.031 \\
\hline $0159+2326$ & 0.0178 & $\mathrm{Sc}+$ & DANS & 14.72 & - & $1646+2725$ & 0.0339 & $\mathrm{Sc}+$ & DHIIH & 17.87 & 0.288 \\
\hline $0159+2354$ & 0.0170 & $\mathrm{Sa}$ & HIIH & 16.07 & 0.565 & $1647+2950$ & 0.0290 & $\mathrm{SBc}+$ & $\mathrm{SBN}$ & 14.68 & 0.736 \\
\hline $1246+2727$ & 0.0199 & - & HIIH & - & 0.775 & $1647+2729$ & 0.0366 & $\mathrm{Sb}$ & $\mathrm{SBN}$ & 15.22 & 0.895 \\
\hline $1247+2701$ & 0.0231 & $\mathrm{Sc}+$ & DANS & 15.97 & 0.515 & $1647+2727$ & 0.0369 & $\mathrm{Sa}$ & SBN & 16.29 & 0.678 \\
\hline $1248+2912$ & 0.0217 & - & SBN & - & 0.715 & $1648+2855$ & 0.0308 & $\mathrm{Sa}$ & HIIH & 14.98 & 0.247 \\
\hline $1253+2756$ & 0.0165 & $\mathrm{Sa}$ & $\mathrm{HIIH}$ & 15.09 & - & $1653+2644$ & 0.0393 & - & $\mathrm{SBN}$ & - & - \\
\hline $1254+2741$ & 0.0172 & $\mathrm{Sb}$ & $\mathrm{SBN}$ & 15.81 & 0.645 & $1654+2812$ & 0.0348 & $\mathrm{Sc}+$ & DHIIH & 17.26 & 0.313 \\
\hline $1254+2802$ & 0.0253 & $\mathrm{Sc}+$ & DANS & 15.76 & - & $1655+2755$ & 0.0349 & $\mathrm{Sb}$ & Sy2 & 14.55 & 0.583 \\
\hline $1255+2819$ & 0.0273 & $\mathrm{Sb}$ & SBN & 15.01 & 0.651 & $1656+2744$ & 0.0330 & $\mathrm{Sa}$ & SBN & 16.37 & 0.578 \\
\hline $1255+3125$ & 0.0258 & $\mathrm{Sa}$ & $\mathrm{HIIH}$ & 15.07 & 0.409 & $1657+2901$ & 0.0317 & $\mathrm{Sc}+$ & DANS & 16.42 & 0.561 \\
\hline
\end{tabular}


Table 1. continued

\begin{tabular}{|c|c|c|c|c|c|c|c|c|c|c|c|}
\hline $\begin{array}{l}\text { UCM name } \\
\text { (1) }\end{array}$ & $\begin{array}{c}z \\
(2)\end{array}$ & $\begin{array}{c}\text { MphT } \\
(3)\end{array}$ & $\begin{array}{l}\text { SpT } \\
(4)\end{array}$ & $\begin{array}{l}m_{\mathrm{r}} \\
(5)\end{array}$ & $\begin{array}{c}E(B-V) \\
(6)\end{array}$ & $\begin{array}{l}\text { UCM name } \\
\text { (1) }\end{array}$ & $\begin{array}{c}z \\
(2)\end{array}$ & $\begin{array}{c}\text { MphT } \\
(3)\end{array}$ & $\begin{array}{c}\text { SpT } \\
(4)\end{array}$ & $\begin{array}{l}m_{\mathrm{r}} \\
(5)\end{array}$ & $\begin{array}{c}E(B-V) \\
(6)\end{array}$ \\
\hline $101659+2928$ & 0.0369 & $\overline{\overline{\text { SB0 }}}$ & 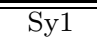 & 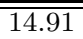 & $\overline{0.528}$ & $202313+2517$ & $\overline{0.0273}$ & $=$ & $\overline{\mathrm{SBN}}$ & $=$ & $=$ \\
\hline $1701+3131$ & 0.0345 & So & Sy1 & 14.44 & 1.904 & $2315+1923$ & 0.0385 & $\mathrm{Sa}$ & HIIH & 16.81 & 0.495 \\
\hline $2238+2308$ & 0.0240 & $\mathrm{Sa}$ & SBN & 14.00 & 1.051 & $2316+2457$ & 0.0277 & $\mathrm{SBa}$ & $\mathrm{SBN}$ & 13.45 & 1.172 \\
\hline $2239+1959$ & 0.0258 & S0 & HIIH & 14.17 & 0.537 & $2316+2459$ & 0.0274 & $\mathrm{Sc}+$ & $\mathrm{SBN}$ & 15.00 & 0.894 \\
\hline $2249+2149$ & 0.0462 & $\mathrm{Sa}$ & SBN & 14.88 & - & $2316+2028$ & 0.0263 & $\mathrm{Sc}+$ & DANS & 16.57 & 0.755 \\
\hline $2250+2427$ & 0.0429 & $\mathrm{Sa}$ & $\mathrm{SBN}$ & 14.78 & 0.773 & $2317+2356$ & 0.0334 & $\mathrm{Sa}$ & SBN & 13.20 & - \\
\hline $2251+2352$ & 0.0267 & $\mathrm{Sc}+$ & DANS & 15.71 & 0.184 & $2319+2234$ & 0.0364 & $\mathrm{Sc}+$ & $\mathrm{SBN}$ & 15.89 & 0.588 \\
\hline $2253+2219$ & 0.0242 & $\mathrm{Sa}$ & SBN & 15.41 & 0.537 & $2319+2243$ & 0.0313 & So & $\mathrm{SBN}$ & 14.75 & - \\
\hline $2255+1930 \mathrm{~S}$ & 0.0203 & $\mathrm{Sb}$ & SBN & 15.42 & 0.493 & $2320+2428$ & 0.0328 & $\mathrm{Sa}$ & DANS & 14.45 & - \\
\hline $2255+1930 \mathrm{~N}$ & 0.0198 & $\mathrm{Sb}$ & $\mathrm{SBN}$ & 14.69 & 0.699 & $2321+2149$ & 0.0374 & $\mathrm{Sc}+$ & $\mathrm{SBN}$ & 15.85 & 0.559 \\
\hline $2255+1926$ & 0.0193 & $\mathrm{Sc}+$ & HIIH & 16.11 & 0.366 & $2321+2506$ & 0.0331 & $\mathrm{Sc}+$ & SBN & 15.26 & - \\
\hline $2255+1654$ & 0.0388 & $\mathrm{Sc}+$ & $\mathrm{SBN}$ & 15.37 & 1.473 & $2322+2218$ & 0.0249 & $\mathrm{Sc}+$ & $\mathrm{SBN}$ & 16.47 & 0.676 \\
\hline $2256+2001$ & 0.0242 & $\mathrm{Sc}+$ & DANS & 14.60 & - & $2324+2448$ & 0.0123 & $\mathrm{Sc}+$ & SBN & 12.75 & 1.300 \\
\hline $2257+2438$ & 0.0345 & S0 & Sy1 & 15.88 & 0.540 & $2325+2318$ & 0.0122 & - & $\mathrm{HIIH}$ & - & - \\
\hline $2257+1606$ & 0.0339 & - & SBN & - & 0.807 & $2325+2208$ & 0.0130 & $\mathrm{SBc}+$ & SBN & 12.09 & - \\
\hline $2258+1920$ & 0.0220 & $\mathrm{Sc}+$ & DANS & 15.42 & 0.348 & $2326+2435$ & 0.0174 & $\mathrm{Sa}$ & DHIIH & 15.87 & 0.278 \\
\hline $2300+2015$ & 0.0346 & $\mathrm{Sb}$ & SBN & 15.60 & 0.326 & $2327+2515 \mathrm{~N}$ & 0.0206 & $\mathrm{Sb}$ & HIIH & 15.59 & 0.474 \\
\hline $2302+2053 \mathrm{~W}$ & 0.0328 & $\mathrm{Sb}$ & HIIH & 16.87 & 0.457 & $2327+2515 \mathrm{~S}$ & 0.0206 & S0 & $\mathrm{HIIH}$ & 15.25 & 0.364 \\
\hline $2302+2053 \mathrm{E}$ & 0.0328 & $\mathrm{Sb}$ & $\mathrm{SBN}$ & 14.69 & 1.301 & $2329+2427$ & 0.0200 & $\mathrm{Sb}$ & DANS & 14.70 & - \\
\hline $2303+1856$ & 0.0276 & $\mathrm{Sa}$ & SBN & 14.73 & 1.199 & $2329+2500$ & 0.0305 & $\mathrm{~S}(\mathrm{r})$ & Sy1 & 15.16 & - \\
\hline $2303+1702$ & 0.0428 & $\mathrm{Sc}+$ & Sy2 & 16.19 & 0.416 & $2329+2512$ & 0.0133 & $\mathrm{Sa}$ & DHIIH & 16.02 & 0.453 \\
\hline $2304+1640$ & 0.0179 & $\mathrm{BCD}$ & BCD & 17.15 & 0.333 & $2331+2214$ & 0.0352 & $\mathrm{Sb}$ & SBN & 16.44 & 0.892 \\
\hline $2304+1621$ & 0.0384 & $\mathrm{Sa}$ & DANS & 15.40 & 0.397 & $2333+2248$ & 0.0399 & $\mathrm{Sc}+$ & $\mathrm{HIIH}$ & 16.37 & 0.383 \\
\hline $2307+1947$ & 0.0271 & $\mathrm{Sb}$ & DANS & 15.56 & 0.453 & $2333+2359$ & 0.0395 & So & Sy1 & 15.84 & 0.197 \\
\hline $2310+1800$ & 0.0363 & $\mathrm{Sc}+$ & $\mathrm{SBN}$ & 15.64 & 0.904 & $2348+2407$ & 0.0359 & $\mathrm{Sa}$ & $\mathrm{SBN}$ & 16.29 & 0.517 \\
\hline $2312+2204$ & 0.0327 & - & SBN & - & 0.864 & $2351+2321$ & 0.0273 & $\mathrm{Sb}$ & HIIH & 16.39 & - \\
\hline $2313+1841$ & 0.0300 & $\mathrm{Sb}$ & SBN & 16.26 & 0.914 & & & & & & \\
\hline
\end{tabular}

Table 1. (1) UCM Survey catalog name as denominated in Zamorano et al. (1994 and 1996) according to their B1950 coordinates. Objects are arranged in order of increasing right ascension. (2) Redshift extracted by Gallego et al. (1996) from emission lines; the mean error value is lower than $310^{-5}$. (3) Hubble morphological type assigned by Vitores et al. (1996a) using five different criteria involving bulge-disk ratios, concentration indexes and mean effective surface brightnesses in the Gunn $r$ band. (4) Spectroscopic type assigned by Gallego et al. (1996) mainly from emission line ratios. (5) Gunn $r$ magnitude from Vitores et al. (1996a); the mean error is 0.08 magnitudes. (6) $B-V$ excess calculated from the Balmer decrement as given by Gallego et al. (1996).

Whereas our sample of galaxies was observed in the Gunn $r$ filter, photometric star data from Landolt (1992) refer to the Cousins system. Therefore, we have corrected the colours included in the Bouguer fit with an averaged $r-R_{\mathrm{C}}=0.37$ (Fukugita et al. 1995).

The errors of the galaxy magnitudes due to the Bouguer fit were calculated for each object with the covariance matrix of the least-square fit according to the expression:

$\Delta m_{\text {Bouguer }}=t_{1 \%} \cdot \sigma_{\mathrm{lsf}} \cdot \sqrt{X^{\dagger} \cdot A^{-1} \cdot X}$ column and line matrixes $X$ and $X^{\dagger}$ for each object are defined as:

$$
\begin{aligned}
& A=\left[\begin{array}{ccc}
N & \sum_{i=1}^{N} X_{i} & \sum_{i=1}^{N}(B-r)_{i} \\
\sum_{i=1}^{N} X_{i} & \sum_{i=1}^{N} X_{i}^{2} & \sum_{i=1}^{N} X_{i} \cdot(B-r)_{i} \\
\sum_{i=1}^{N}(B-r)_{i} & \sum_{i=1}^{N} X_{i} \cdot(B-r)_{i} & \sum_{i=1}^{N}(B-r)_{i}^{2}
\end{array}\right] \\
& X=\left(\begin{array}{c}
1 \\
X \\
B-r
\end{array}\right) \\
& X X^{\dagger}=\left(\begin{array}{lll}
1 & X & B-r
\end{array}\right) .
\end{aligned}
$$

The transformation equations for each night are listed in Table 2.

\subsection{Galaxy integrated photometry}

Many galaxies were found to be very irregular in shape, being very difficult to apply the standard circular of the standard deviation of the least-square fit. The variance-covariance matrix of the least-square fit, $A$, the 
Table 2. Instrument features and photometric transformations for each night

\begin{tabular}{|c|c|c|c|c|c|c|c|c|}
\hline Telescope & Date & $\begin{array}{c}\text { CCD } \\
(3) \\
\end{array}$ & $\begin{array}{c}\mathrm{RN} \\
\left(\mathrm{e}^{-}\right) \\
(4) \\
\end{array}$ & $\begin{array}{c}\text { Gain } \\
\left(\mathrm{e}^{-} / \mathrm{ADU}\right) \\
(5) \\
\end{array}$ & $\begin{array}{l}\text { Scale } \\
\left({ }^{\prime \prime} / \text { pix }\right) \\
(6)\end{array}$ & $\begin{array}{l}C \\
(7) \\
\end{array}$ & $\begin{array}{r}K_{B} \\
(8) \\
\end{array}$ & $\begin{array}{c}K_{B-r} \\
(9) \\
\end{array}$ \\
\hline JKT & "Nov. 27, 1997 & $\overline{\text { TEK\#4 }}$ & 4.10 & $\overline{1.63}$ & 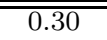 & $23.00 \pm 0.03$ & $=-0.22 \pm 0.02$ & $\overline{0.03 \pm 0.01}$ \\
\hline JKT & Dec. 01, 1997 & TEK\#4 & 4.10 & 1.63 & 0.30 & $23.01 \pm 0.07$ & $-0.24 \pm 0.05$ & $0.02 \pm 0.01$ \\
\hline JKT & Dec. 02, 1997 & TEK\#4 & 4.10 & 1.63 & 0.30 & $22.78 \pm 0.08$ & $-0.12 \pm 0.06$ & $0.01 \pm 0.02$ \\
\hline $1.52 \mathrm{~m}$ & Jun. 18, 1998 & TEK1024 & 6.38 & 6.55 & 0.40 & $21.45 \pm 0.03$ & $-0.33 \pm 0.02$ & $0.09 \pm 0.01$ \\
\hline $1.52 \mathrm{~m}$ & Jun. 19, 1998 & TEK1024 & 6.38 & 6.55 & 0.40 & $21.40 \pm 0.06$ & $-0.27 \pm 0.04$ & $0.09 \pm 0.01$ \\
\hline $1.23 \mathrm{~m}$ & Oct. 28,1998 & TEK7c_12 & 5.52 & 0.80 & 0.50 & $22.28 \pm 0.02$ & $-0.21 \pm 0.01$ & $0.08 \pm 0.01$ \\
\hline $1.52 \mathrm{~m}$ & Jun. 10, 1999 & TEK1024 & 6.38 & 6.55 & 0.40 & $21.80 \pm 0.14$ & $-0.36 \pm 0.10$ & $0.10 \pm 0.03$ \\
\hline $1.23 \mathrm{~m}$ & Jun. 16, 1999 & LORAL\#11 & 8.50 & 1.70 & 0.31 & $22.95 \pm 0.06$ & $-0.27 \pm 0.04$ & $0.04 \pm 0.01$ \\
\hline $1.23 \mathrm{~m}$ & Jun. 17, 1999 & SITe\#18 & 5.20 & 2.60 & 0.50 & $22.03 \pm 0.03$ & $-0.19 \pm 0.02$ & $0.05 \pm 0.01$ \\
\hline $1.23 \mathrm{~m}$ & Jun. 19, 1999 & TEK\#13 & 5.10 & 0.60 & 0.50 & $22.81 \pm 0.04$ & $-0.25 \pm 0.03$ & $0.09 \pm 0.01$ \\
\hline $1.23 \mathrm{~m}$ & Jun. 20, 1999 & TEK\#13 & 5.10 & 0.60 & 0.50 & $22.84 \pm 0.05$ & $-0.25 \pm 0.03$ & $0.07 \pm 0.01$ \\
\hline JKT & Jul. 12, 1999 & TEK\#5 & 4.82 & 1.53 & 0.30 & $23.09 \pm 0.03$ & $-0.50 \pm 0.02$ & $0.04 \pm 0.01$ \\
\hline JKT & Jul. 13, 1999 & TEK\#5 & 4.82 & 1.53 & 0.30 & $22.77 \pm 0.05$ & $-0.21 \pm 0.04$ & $0.03 \pm 0.01$ \\
\hline JKT & Jul. 15, 1999 & TEK\#5 & 4.82 & 1.53 & 0.30 & $22.81 \pm 0.02$ & $-0.24 \pm 0.01$ & $0.07 \pm 0.01$ \\
\hline JKT & Jul. 16, 1999 & TEK\#5 & 4.82 & 1.53 & 0.30 & $22.75 \pm 0.03$ & $-0.25 \pm 0.03$ & $0.07 \pm 0.01$ \\
\hline JKT & Jul. 17, 1999 & TEK\#5 & 4.82 & 1.53 & 0.30 & $22.75 \pm 0.01$ & $-0.20 \pm 0.01$ & $0.06 \pm 0.01$ \\
\hline JKT & Jul. 18, 1999 & TEK\# $\#$ & 4.82 & 1.53 & 0.30 & $22.85 \pm 0.05$ & $-0.27 \pm 0.04$ & $0.06 \pm 0.01$ \\
\hline
\end{tabular}

Table 2. (1) Telescope name. JKT stands for the Jacobus Kapteyn Telescope in La Palma (Spain); $1.52 \mathrm{~m}$ for the Spanish Telescope in Calar Alto, Almería (Spain); $1.23 \mathrm{~m}$ refers to the telescope at the German-Spanish Observatory in Calar Alto. (2) Date of the observation. (3) CCD detector used. (4) Readout noise of the CCD in electrons. (5) Gain of the CCD in electrons per ADU. (6) Scale of the chip in arcsec per pixel. (7) Instrumental constant of the photometric calibration for each night using Landolt (1992) stars. (8) Extinction in the Johnson $B$ band. (9) Colour term of the Bouguer fit refered to the $B-r$ colour (Johnson $B$ and Gunn $r$ ).

apertures. We decided to measure fluxes using the IRAF task polyphot. This task allowed us to build polygons around the galaxies including the whole object and minimizing the area of sky also included. At least two polygons were used in three different positions (securing a minimum of six measures) to avoid errors due to the specific shape of the polygon. The sky was determined as an average of at least 8 measures with a circular aperture around the object.

The errors were calculated as follows. Each flux measurement included an error due to Poisson noise, the uncertainty in the sky determination, and the readout noise of the CCD. This error, in magnitude representation, is described by the expression:

$\Delta m_{i}=1.0857 \cdot \frac{\sqrt{\frac{F}{G}+\text { Area } \cdot \sigma_{\mathrm{sky}}^{2}+\frac{\mathrm{Area}^{2} \cdot \sigma_{\mathrm{sky}}^{2}}{N_{\mathrm{sky}}}}}{F}$

where $F$ is the flux in counts $\mathrm{s}^{-1}, G$ is the CCD gain in counts $\mathrm{e}^{-1}$, Area is the area in pixels enclosed by the polygon, $\sigma_{\text {sky }}$ is the standard deviation of the sky measure and $N_{\text {sky }}$ is the number of pixels of the sky measure. The first term of the sum inside the square root is the Poisson noise (square root of the number of electrons counted), the second term refers to the uncertainty in the determination of the sky level, and the third is related to the effects of flatfield errors in the sky determination.

Several polygon measures were taken to assure a good magnitude determination. The final associated error was chosen to be the greatest among all the associated to each polygon and the standard deviation of all the polygon measures:

$\Delta m_{\text {Flux }}=\max \left(\Delta m_{i}\right)$.

Finally the Bouguer line errors were also taken in consideration, yielding a final expression for the magnitude error:

$\Delta m_{B}=\sqrt{\left(\Delta m_{\text {Bouguer }}\right)^{2}+\left(\Delta m_{\text {Flux }}\right)^{2}}$.

Apparent total $B$ magnitudes, as measured with this method, are listed in Table 3.

We have also calculated the $B$ magnitudes inside the $24 \mathrm{mag} \operatorname{arcsec}^{-2}$ isophote $\left(B_{24}\right)$, and the total magnitudes using the Kron (1980) radius defined as:

$r_{k}=\frac{\sum_{i} r_{i} \cdot F_{i}}{\sum_{i} F_{i}}$

where $i$ runs from the center to the aperture which has an isophotal level corresponding to the standard deviation of the sky. A second set of total magnitudes were measured within an aperture of radius $2 \cdot r_{k}$ applying this method. In average, Kron magnitudes were $0.02^{\mathrm{m}}$ fainter than the polygonal ones; the absolute differences ranged from 0.00 to 0.47 magnitudes. The highest differences were always due to the presence of field stars inside the Kron aperture or flux contamination from nearby objects, which have been previously deleted interactively using the CR_UTILS IRAF package. 
The apparent magnitudes were converted into absolute magnitudes using the redshifts listed in Table 1 . The standard galactic extinction correction was applied using the Burstein \& Heiles (1982) maps. Because the Balmer decrements are also available for most of the objects (Gallego et al. 1996), we provide these values in Table 1 to allow the correction from total extinction (Galactic and internal) through the $B-V$ colour excess.

\subsection{Effective radii and colours}

The effective radius (defined as the radius that contains half of the total light) in the $B$ images was measured in two different ways. First, an equivalent half light radius in arcsec was calculated as the geometric mean of the major and minor semi-axes of the elliptical isophote containing half of the galaxy flux (i.e., $B_{\mathrm{T}}+0.75$ magnitudes); this half-light radius $r_{1 / 2}\left({ }^{\prime \prime}\right)$ is tabulated in Col. (5) of Table 3. We also measured the flux of the galaxy inside circular apertures and selected the one containing half of the light. These radii were transformed into effective radius in $\mathrm{kpc}$ $\left(R_{\mathrm{e}}\right.$, Col. (4) of Table 3 ) with the formula:

$R_{\mathrm{e}}(\mathrm{kpc})=58.1 \cdot r_{\mathrm{e}}\left({ }^{\prime \prime}\right) \cdot \frac{\left[(1+z)(1+z)^{0.5}\right]}{(1+z)^{2}}$.

$B-r$ colours have also been calculated. We first aligned the Johnson $B$ images with the original Gunn $r$ images from Vitores et al. (1996a). Permitted modifications were rotation, scaling and shift. We measured the aperture colour inside the $24 \mathrm{mag} \operatorname{arcsec}^{-2}$ Johnson $B$ isophote. Then we also obtained the colour inside the isophote of radius the effective radius (as measured in the $B$ band). Again, the Galactic extinction correction was performed using the Burstein \& Heiles (1982) maps. Conversion constants are 3.98 in $B$ and 2.51 in $r$; both values were interpolated from Fitzpatrick (1999).

In Table 3 we summarize all these results: apparent total and $B_{24}$ magnitudes in Cols. (2) and (3); effective radius in kpc and arcsec in Cols. (4) and (5) respectively; absolute $B$ magnitudes corrected from Galactic extinction in Col. (6) and effective and isophote $24 \mathrm{mag} \operatorname{arcsec}^{-2}$ $B-r$ colours in Cols. (7) and (8). Colour information is only available for those galaxies with Gunn $r$ magnitude measured by Vitores et al. (1996a).

\section{Data analysis}

In Fig. 1 we plot the Gunn $r$ and Johnson $B$ total apparent magnitude histograms of the UCM Survey galaxies. They were arranged in 0.5 magnitude bins. Both distributions cover a range of about seven magnitudes and present a rather symmetric shape around $16.5^{\mathrm{m}}$ in the $B$ bandpass and $16.0^{\mathrm{m}}$ in the $r$ filter. The average of the Johnson $B$ distribution is $16.1 \pm 1.1$. In the Gunn $r$ filter the average is $15.5 \pm 1.0$. These values are plotted at the top of the
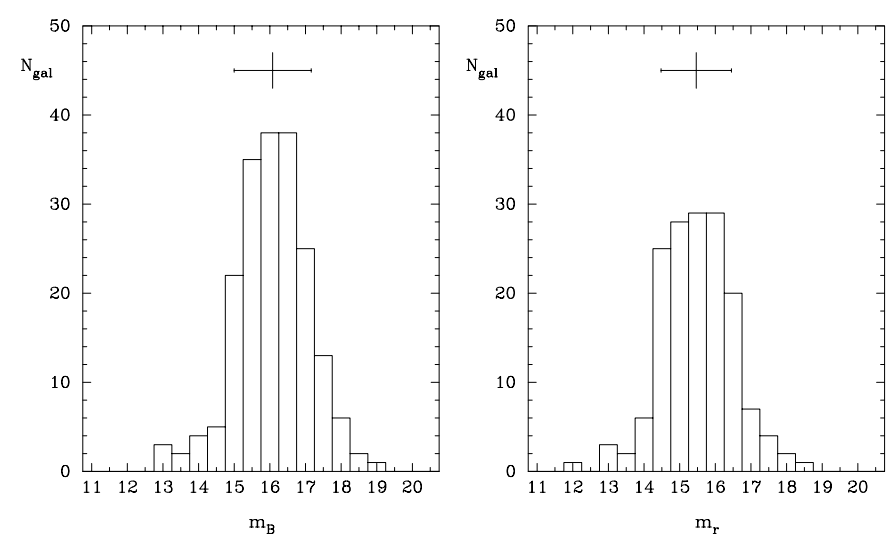

Fig. 1. Johnson $B$ and Gunn $r$ histograms of the UCM Survey. The top error bar shows the average and the standard deviation of the data (see text). The average colour results 0.71 magnitudes. The Gunn $r$ data have been extracted from Vitores et al. (1996a)

diagram. Both histograms show a sharp bright magnitude cutoff (around 14.5-15.0 in the $B$-band and 13.75-14.25 in the $r$ band) due to detection problems (the objectiveprism spectra of very bright objects are saturated, not allowing the detection of the emission lines); there is also a faint magnitude limit around 19 magnitudes in the blue filter and 18 in the red one.

We plot the absolute total magnitudes versus the effective radii of the UCM galaxies in Fig. 2. Galaxies were labelled depending of their spectroscopic type (see Gallego et al. 1996 for details):

SBN - Starburst Nuclei - Originally defined by Balzano (1983), they show high extinction values, with very low $[\mathrm{NII}] / \mathrm{H} \alpha$ ratios and faint [OIII $] \lambda 5007$ emission. Their $\mathrm{H} \alpha$ luminosities are always higher than $10^{8} \mathrm{~L} \odot$.

DANS - Dwarf Amorphous Nuclear Starburst Introduced by Salzer et al. (1989), they show very similar spectroscopic properties to SBN objects, but with $\mathrm{H} \alpha$ luminosities lower than $510^{7} \mathrm{~L} \odot$.

HIIH - HII Hotspot - The HII Hotspot class shows similar $\mathrm{H} \alpha$ luminosities to those measured in SBN galaxies but with large $[\mathrm{OIII}] \lambda 5007 / \mathrm{H} \beta$ ratios, that is, higher ionization.

DHIIH - Dwarf HII Hotspot - This is an HIIH subclass with identical spectroscopic properties but $\mathrm{H} \alpha$ luminosities lower than $510^{7} L_{\odot}$.

BCD - Blue Compact Dwarf - The lowest luminosity and highest ionization objects have been classified as Blue Compact Dwarf galaxies, showing in all cases $\mathrm{H} \alpha$ luminosities lower than $510^{7} L \odot$. They also show large [OIII $] \lambda 5007 / \mathrm{H} \beta$ and $\mathrm{H} \alpha /[\mathrm{NII}] \lambda 6584$ line ratios and intense $[\mathrm{OII}] \lambda 3727$ emission.

All these spectroscopic classes are usually collapsed in two main categories: starburst disk-like (SB hereafter) and HII-like galaxies (see Guzmán et al. 1997; Gallego 1998). The SB-like class includes SBN and DANS spectroscopic 
Table 3. Photometry results in the $B$ and $r$ bandpass for the UCM survey

\begin{tabular}{|c|c|c|c|c|c|c|c|}
\hline $\begin{array}{l}\text { UCM name } \\
\text { (1) }\end{array}$ & $\begin{array}{c}\left(m_{B}\right)_{\mathrm{T}} \\
(2)\end{array}$ & $\begin{array}{c}\left(m_{B}\right)_{24} \\
(3)\end{array}$ & $\begin{array}{c}R_{\mathrm{e}}(\mathrm{kpc}) \\
(4)\end{array}$ & $\begin{array}{c}r_{1 / 2}\left({ }^{\prime \prime}\right) \\
(5)\end{array}$ & $\begin{array}{c}M_{B} \\
(6)\end{array}$ & $\begin{array}{c}(B-r)_{\text {ef }} \\
(7)\end{array}$ & $\begin{array}{c}(B-r)_{24} \\
(8)\end{array}$ \\
\hline 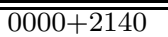 & 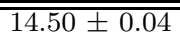 & 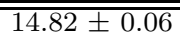 & 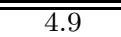 & 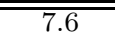 & $=-21.41 \pm 0.05$ & ב- & $=$ \\
\hline $0003+2200$ & $17.64 \pm 0.05$ & $17.81 \pm 0.10$ & 2.1 & 5.3 & $-18.16 \pm 0.07$ & $1.37 \pm 0.11$ & $1.37 \pm 0.14$ \\
\hline $0003+2215$ & $16.63 \pm 0.05$ & $16.98 \pm 0.08$ & 4.5 & 9.6 & $-19.15 \pm 0.06$ & - & - \\
\hline $0003+1955$ & $14.09 \pm 0.04$ & $14.12 \pm 0.07$ & 0.8 & 1.0 & $-22.14 \pm 0.06$ & - & - \\
\hline $0005+1802$ & $16.32 \pm 0.06$ & $16.52 \pm 0.08$ & 2.1 & 3.8 & $-19.03 \pm 0.08$ & - & - \\
\hline $0006+2332$ & $14.92 \pm 0.02$ & $15.11 \pm 0.05$ & 4.1 & 8.5 & $-20.18 \pm 0.05$ & 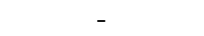 & - \\
\hline $0013+1942$ & $17.11 \pm 0.06$ & $17.32 \pm 0.08$ & 2.0 & 2.7 & $-19.04 \pm 0.07$ & $-0.06 \pm 0.06$ & $0.52 \pm 0.09$ \\
\hline $0014+1829$ & $16.09 \pm 0.10$ & $16.31 \pm 0.08$ & 1.6 & 3.2 & $-19.21 \pm 0.11$ & $0.55 \pm 0.12$ & $0.58 \pm 0.13$ \\
\hline $0014+1748$ & $14.87 \pm 0.03$ & $15.21 \pm 0.06$ & 7.9 & 13.6 & $-20.41 \pm 0.05$ & $1.15 \pm 0.10$ & $1.06 \pm 0.12$ \\
\hline $0015+2212$ & $16.54 \pm 0.04$ & $16.83 \pm 0.07$ & 1.3 & 2.6 & $-18.97 \pm 0.06$ & $0.53 \pm 0.33$ & $0.84 \pm 0.34$ \\
\hline $0017+1942$ & $15.83 \pm 0.04$ & $15.97 \pm 0.06$ & 4.3 & 4.5 & $-20.39 \pm 0.05$ & $0.53 \pm 0.11$ & $0.52 \pm 0.12$ \\
\hline $0017+2148$ & $16.69 \pm 0.05$ & $17.07 \pm 0.09$ & 1.1 & 2.7 & $-18.74 \pm 0.07$ & - & - \\
\hline $0018+2216$ & $16.83 \pm 0.01$ & $16.91 \pm 0.03$ & 1.1 & 2.2 & $-18.34 \pm 0.05$ & $0.56 \pm 0.03$ & $0.79 \pm 0.05$ \\
\hline $0018+2218$ & $15.80 \pm 0.03$ & $16.24 \pm 0.04$ & 6.2 & 13.1 & $-19.95 \pm 0.05$ & & - \\
\hline $0019+2201$ & $16.47 \pm 0.03$ & $16.87 \pm 0.05$ & 2.5 & 4.5 & $-18.97 \pm 0.05$ & $1.11 \pm 0.33$ & $1.08 \pm 0.34$ \\
\hline $0022+2049$ & $15.62 \pm 0.02$ & $15.76 \pm 0.06$ & 2.3 & 4.1 & $-19.73 \pm 0.05$ & $1.16 \pm 0.10$ & $1.16 \pm 0.11$ \\
\hline $0023+1908$ & $16.78 \pm 0.04$ & $16.89 \pm 0.18$ & 1.5 & 2.2 & $-19.23 \pm 0.05$ & - & - \\
\hline $0034+2119$ & $15.80 \pm 0.04$ & $16.09 \pm 0.08$ & 5.3 & 5.5 & $-20.66 \pm 0.06$ & - & - \\
\hline $0037+2226$ & $14.57 \pm 0.02$ & $14.71 \pm 0.07$ & 4.9 & 9.8 & $-20.95 \pm 0.05$ & - & - \\
\hline $0038+2259$ & $16.15 \pm 0.04$ & $16.32 \pm 0.06$ & 7.5 & 5.5 & $-21.14 \pm 0.05$ & $1.28 \pm 0.10$ & $1.25 \pm 0.11$ \\
\hline $0039+0054$ & $14.91 \pm 0.09$ & $15.29 \pm 0.08$ & 6.7 & 15.6 & $-20.40 \pm 0.10$ & - & - \\
\hline $0040+0257$ & $16.84 \pm 0.05$ & $17.02 \pm 0.13$ & 2.3 & 2.1 & $-19.95 \pm 0.06$ & $-0.25 \pm 0.03$ & $0.10 \pm 0.13$ \\
\hline $0040+2312$ & $15.59 \pm 0.03$ & $15.96 \pm 0.05$ & 6.8 & 7.6 & $-20.38 \pm 0.05$ & - & - \\
\hline $0040+0220$ & $17.07 \pm 0.02$ & $17.21 \pm 0.07$ & 1.0 & 2.0 & $-18.04 \pm 0.05$ & $0.44 \pm 0.10$ & $0.67 \pm 0.12$ \\
\hline $0040-0023$ & $13.64 \pm 0.02$ & $13.87 \pm 0.03$ & 5.8 & 14.9 & $-21.04 \pm 0.06$ & - & - \\
\hline $0041+0134$ & $14.31 \pm 0.02$ & $14.63 \pm 0.05$ & 9.9 & 21.0 & $-20.76 \pm 0.05$ & - & - \\
\hline $0043+0245$ & $17.24 \pm 0.09$ & $17.36 \pm 0.14$ & 1.0 & 2.0 & $-18.03 \pm 0.10$ & - & - \\
\hline 0043-0159 & $13.05 \pm 0.01$ & $13.09 \pm 0.07$ & 8.1 & 17.1 & $-21.94 \pm 0.05$ & - & - \\
\hline $0044+2246$ & $15.97 \pm 0.02$ & $16.26 \pm 0.06$ & 5.7 & 7.3 & $-20.04 \pm 0.05$ & $1.20 \pm 0.15$ & $1.08 \pm 0.16$ \\
\hline $0045+2206$ & $14.97 \pm 0.03$ & $15.08 \pm 0.06$ & 1.9 & 3.9 & $-20.53 \pm 0.05$ & - & - \\
\hline $0047+2051$ & $16.86 \pm 0.02$ & $16.91 \pm 0.08$ & 4.0 & 2.9 & $-20.94 \pm 0.04$ & $0.60 \pm 0.10$ & $0.77 \pm 0.12$ \\
\hline $0047-0213$ & $15.53 \pm 0.03$ & $15.71 \pm 0.09$ & 1.4 & 3.8 & $-19.32 \pm 0.06$ & $0.49 \pm 0.03$ & $0.67 \pm 0.10$ \\
\hline $0047+2413$ & $15.72 \pm 0.03$ & $15.96 \pm 0.09$ & 7.3 & 6.7 & $-20.99 \pm 0.05$ & $1.07 \pm 0.05$ & $1.02 \pm 0.10$ \\
\hline $0047+2414$ & $15.21 \pm 0.03$ & $15.28 \pm 0.07$ & 5.1 & 4.9 & $-21.50 \pm 0.05$ & - & - \\
\hline 0049-0006 & $18.24 \pm 0.13$ & $18.77 \pm 0.13$ & 1.9 & 1.7 & $-18.60 \pm 0.14$ & $0.01 \pm 0.17^{\dagger}$ & - \\
\hline $0049+0017$ & $16.97 \pm 0.02$ & $17.38 \pm 0.07$ & 1.4 & 3.7 & $-17.71 \pm 0.06$ & $-0.33 \pm 0.04$ & $0.16 \pm 0.08$ \\
\hline $0049-0045$ & $15.21 \pm 0.01$ & $15.39 \pm 0.05$ & 0.7 & 5.7 & $-17.23 \pm 0.14$ & - & - \\
\hline $0050+0005$ & $16.26 \pm 0.02$ & $16.46 \pm 0.06$ & 2.9 & 3.0 & $-20.40 \pm 0.04$ & $0.44 \pm 0.05$ & $0.50 \pm 0.07$ \\
\hline $0050+2114$ & $15.53 \pm 0.06$ & - & - & - & $-20.41 \pm 0.07$ & $0.83 \pm 0.33^{\dagger}$ & - \\
\hline $0051+2430$ & $15.19 \pm 0.04$ & $15.34 \pm 0.05$ & 3.8 & 8.5 & $-19.99 \pm 0.07$ & - & - \\
\hline 0054-0133 & $15.74 \pm 0.05$ & $16.09 \pm 0.11$ & 7.2 & 5.7 & $-21.87 \pm 0.06$ & - & - \\
\hline $0054+2337$ & $15.19 \pm 0.02$ & $15.50 \pm 0.05$ & 3.8 & 8.7 & $-19.94 \pm 0.06$ & - & - \\
\hline $0056+0044$ & $16.60 \pm 0.05$ & $17.32 \pm 0.11$ & 3.7 & 10.7 & $-18.67 \pm 0.07$ & $0.28 \pm 0.08$ & $0.26 \pm 0.11$ \\
\hline $0056+0043$ & $16.56 \pm 0.03$ & $16.64 \pm 0.08$ & 1.3 & 2.3 & $-18.78 \pm 0.05$ & $0.26 \pm 0.03$ & $0.42 \pm 0.09$ \\
\hline $0119+2156$ & $16.59 \pm 0.05$ & $16.82 \pm 0.09$ & 9.6 & 5.3 & $-21.32 \pm 0.06$ & $1.26 \pm 0.05$ & $1.13 \pm 0.10$ \\
\hline $0121+2137$ & $15.81 \pm 0.09$ & $15.98 \pm 0.23$ & 8.5 & 9.8 & $-20.93 \pm 0.10$ & $0.51 \pm 0.04$ & $0.40 \pm 0.23$ \\
\hline $0129+2109$ & $15.11 \pm 0.03$ & $15.22 \pm 0.07$ & 8.3 & 10.1 & $-21.66 \pm 0.05$ & - & - \\
\hline $0134+2257$ & $15.89 \pm 0.05$ & $16.26 \pm 0.07$ & 6.9 & 7.3 & $-21.14 \pm 0.06$ & 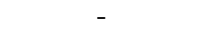 & - \\
\hline $0135+2242$ & $16.79 \pm 0.04$ & $17.21 \pm 0.10$ & 2.4 & 3.0 & $-20.33 \pm 0.05$ & $0.67 \pm 0.04$ & $0.74 \pm 0.11$ \\
\hline $0138+2216$ & $17.58 \pm 0.02$ & $17.82 \pm 0.06$ & 3.9 & 2.3 & $-20.62 \pm 0.04$ & 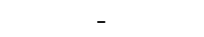 & - \\
\hline $0141+2220$ & $16.26 \pm 0.04$ & $16.36 \pm 0.09$ & 1.7 & 3.0 & $-19.18 \pm 0.06$ & $0.39 \pm 0.09$ & $0.37 \pm 0.13$ \\
\hline $0142+2137$ & $15.39 \pm 0.05$ & $15.66 \pm 0.07$ & 9.4 & 9.9 & $-21.59 \pm 0.06$ & $1.20 \pm 0.10$ & $1.11 \pm 0.12$ \\
\hline $0144+2519$ & $15.64 \pm 0.03$ & $15.89 \pm 0.09$ & 9.6 & 10.7 & $-21.79 \pm 0.05$ & $0.77 \pm 0.10$ & $0.67 \pm 0.13$ \\
\hline $0147+2309$ & $16.72 \pm 0.05$ & $16.94 \pm 0.08$ & 1.9 & 3.4 & $-18.91 \pm 0.07$ & $0.75 \pm 0.10$ & $0.79 \pm 0.13$ \\
\hline $0148+2124$ & $16.88 \pm 0.06$ & $17.28 \pm 0.10$ & 1.2 & 3.2 & $-18.40 \pm 0.08$ & $0.39 \pm 0.11$ & $0.62 \pm 0.14$ \\
\hline $0150+2032$ & $16.66 \pm 0.05$ & $16.99 \pm 0.12$ & 6.3 & 8.7 & $-19.98 \pm 0.07$ & $0.60 \pm 0.15$ & $0.58 \pm 0.16$ \\
\hline $0156+2410$ & $15.16 \pm 0.03$ & $15.33 \pm 0.09$ & 2.1 & 5.3 & $-19.75 \pm 0.06$ & $0.48 \pm 0.03$ & $0.53 \pm 0.10$ \\
\hline $0157+2413$ & $15.03 \pm 0.04$ & $15.16 \pm 0.06$ & 5.4 & 8.7 & $-20.44 \pm 0.06$ & $1.20 \pm 0.04$ & $1.14 \pm 0.07$ \\
\hline $0157+2102$ & $14.87 \pm 0.02$ & $14.95 \pm 0.07$ & 1.6 & 4.4 & $-19.40 \pm 0.07$ & $0.24 \pm 0.03$ & $0.33 \pm 0.08$ \\
\hline $0159+2354$ & $17.19 \pm 0.07$ & $17.41 \pm 0.16$ & 1.1 & 2.4 & $-18.20 \pm 0.09$ & $1.00 \pm 0.13^{\dagger}$ & 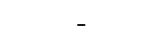 \\
\hline $0159+2326$ & $15.87 \pm 0.02$ & $16.01 \pm 0.05$ & 2.5 & 4.9 & $-19.56 \pm 0.05$ & $0.99 \pm 0.03$ & $1.02 \pm 0.06$ \\
\hline $1246+2727$ & $15.88 \pm 0.02$ & $15.94 \pm 0.09$ & 3.5 & 5.6 & $-19.55 \pm 0.05$ & 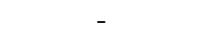 & - \\
\hline $1247+2701$ & $16.63 \pm 0.05$ & $16.77 \pm 0.06$ & 2.3 & 3.4 & $-19.11 \pm 0.07$ & $0.49 \pm 0.04$ & $0.55 \pm 0.07$ \\
\hline $1248+2912$ & $14.87 \pm 0.02$ & $15.18 \pm 0.06$ & 5.8 & 10.5 & $-20.75 \pm 0.05$ & & - \\
\hline $1253+2756$ & $15.81 \pm 0.04$ & $15.98 \pm 0.06$ & 1.5 & 3.2 & $-19.20 \pm 0.06$ & $0.67 \pm 0.10$ & $0.68 \pm 0.10$ \\
\hline $1254+2741$ & $16.70 \pm 0.07$ & $17.20 \pm 0.10$ & 2.4 & 5.0 & $-18.40 \pm 0.08$ & $1.10 \pm 0.07$ & $1.05 \pm 0.10$ \\
\hline
\end{tabular}


Table 3. continued

\begin{tabular}{|c|c|c|c|c|c|c|c|}
\hline $\begin{array}{l}\text { UCM name } \\
(1)\end{array}$ & $\begin{array}{c}\left(m_{B}\right)_{\mathrm{T}} \\
(2)\end{array}$ & $\begin{array}{c}\left(m_{B}\right)_{24} \\
(3)\end{array}$ & $\begin{array}{c}R_{\mathrm{e}}(\mathrm{kpc}) \\
(4)\end{array}$ & $\begin{array}{c}r_{1 / 2}\left({ }^{\prime \prime}\right) \\
(5)\end{array}$ & $\begin{array}{c}M_{B} \\
(6)\end{array}$ & $\begin{array}{c}(B-r)_{\text {ef }} \\
(7)\end{array}$ & $\begin{array}{c}(B-r)_{24} \\
(8)\end{array}$ \\
\hline 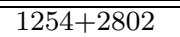 & $=16.81 \pm 0.03$ & 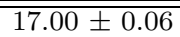 & $\overline{2.9}$ & $\begin{array}{ll}3.7 \\
\end{array}$ & $-19.15 \pm 0.05$ & $0.90 \pm 0.05$ & $0.98 \pm 0.07$ \\
\hline $1255+2819$ & $15.51 \pm 0.07$ & $16.08 \pm 0.08$ & 7.7 & 10.8 & $-20.64 \pm 0.08$ & $0.83 \pm 0.14$ & $0.77 \pm 0.15$ \\
\hline $1255+3125$ & $16.14 \pm 0.08$ & $16.41 \pm 0.08$ & 2.1 & 3.2 & $-19.86 \pm 0.09$ & $1.12 \pm 0.09$ & $1.14 \pm 0.11$ \\
\hline $1255+2734$ & $16.69 \pm 0.02$ & $16.96 \pm 0.06$ & 2.7 & 5.7 & $-19.08 \pm 0.05$ & $0.88 \pm 0.20$ & $0.85 \pm 0.21$ \\
\hline $1256+2717$ & $17.62 \pm 0.07$ & $18.13 \pm 0.09$ & 1.5 & 2.6 & $-18.48 \pm 0.08$ & & 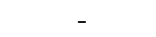 \\
\hline $1256+2732$ & $15.95 \pm 0.06$ & $16.18 \pm 0.06$ & 2.8 & 4.3 & $-19.82 \pm 0.07$ & $0.52 \pm 0.08$ & $0.53 \pm 0.09$ \\
\hline $1256+2701$ & $16.62 \pm 0.05$ & $16.88 \pm 0.08$ & 4.7 & 5.6 & $-19.25 \pm 0.07$ & $0.44 \pm 0.09$ & $0.39 \pm 0.11$ \\
\hline $1256+2910$ & $16.22 \pm 0.05$ & $16.22 \pm 0.05$ & 4.6 & 4.8 & $-19.96 \pm 0.07$ & $0.83 \pm 0.04$ & $0.86 \pm 0.06$ \\
\hline $1256+2823$ & $15.72 \pm 0.12$ & $16.04 \pm 0.15$ & 6.0 & 6.3 & $-20.68 \pm 0.12$ & $0.90 \pm 0.15$ & $0.80 \pm 0.15$ \\
\hline $1256+2754$ & $15.37 \pm 0.05$ & $15.44 \pm 0.06$ & 2.6 & 5.0 & $-19.74 \pm 0.07$ & $0.49 \pm 0.21$ & $0.50 \pm 0.21$ \\
\hline $1256+2722$ & $17.09 \pm 0.05$ & $17.28 \pm 0.10$ & 2.4 & 2.7 & $-19.11 \pm 0.06$ & $0.64 \pm 0.10$ & $0.80 \pm 0.14$ \\
\hline $1257+2808$ & $16.14 \pm 0.02$ & $16.34 \pm 0.04$ & 1.7 & 3.3 & $-19.10 \pm 0.05$ & $0.66 \pm 0.06$ & $0.71 \pm 0.08$ \\
\hline $1258+2754$ & $15.82 \pm 0.04$ & $16.03 \pm 0.06$ & 4.1 & 5.6 & $-20.13 \pm 0.06$ & $0.42 \pm 0.08$ & $0.38 \pm 0.09$ \\
\hline $1259+2934$ & $14.21 \pm 0.04$ & - & - & - & $-21.59 \pm 0.06$ & $0.03 \pm 0.06^{\dagger}$ & - \\
\hline $1259+3011$ & $16.21 \pm 0.04$ & $16.32 \pm 0.07$ & 1.9 & 2.3 & $-20.13 \pm 0.05$ & $0.72 \pm 0.09$ & $0.84 \pm 0.11$ \\
\hline $1259+2755$ & $15.37 \pm 0.02$ & $15.51 \pm 0.05$ & 3.2 & 4.4 & $-20.42 \pm 0.05$ & $0.84 \pm 0.12$ & $0.95 \pm 0.13$ \\
\hline $1300+2907$ & $17.07 \pm 0.04$ & $17.37 \pm 0.12$ & 1.4 & 2.7 & $-18.56 \pm 0.06$ & $0.03 \pm 0.05$ & $0.35 \pm 0.13$ \\
\hline $1301+2904$ & $15.45 \pm 0.13$ & $15.81 \pm 0.14$ & 5.8 & 8.0 & $-20.61 \pm 0.13$ & $0.48 \pm 0.14$ & $0.36 \pm 0.15$ \\
\hline $1302+2853$ & $16.22 \pm 0.02$ & $16.43 \pm 0.04$ & 2.7 & 4.3 & $-19.59 \pm 0.05$ & $0.48 \pm 0.03$ & $0.47 \pm 0.05$ \\
\hline $1302+3032$ & $16.56 \pm 0.02$ & $16.74 \pm 0.05$ & 2.3 & 2.7 & $-20.04 \pm 0.04$ & & 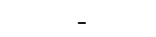 \\
\hline $1303+2908$ & $16.78 \pm 0.03$ & $16.99 \pm 0.12$ & 3.5 & 4.6 & $-19.24 \pm 0.05$ & $0.38 \pm 0.13$ & $0.56 \pm 0.14$ \\
\hline $1304+2808$ & $15.84 \pm 0.07$ & $16.12 \pm 0.10$ & 4.2 & 8.8 & $-19.71 \pm 0.08$ & $1.12 \pm 0.12$ & $1.04 \pm 0.13$ \\
\hline $1304+2830$ & $18.57 \pm 0.05$ & $18.69 \pm 0.05$ & 0.8 & 1.3 & $-17.05 \pm 0.07$ & $-0.08 \pm 0.03$ & $0.41 \pm 0.07$ \\
\hline $1304+2907$ & $15.12 \pm 0.07$ & $15.38 \pm 0.10$ & 5.1 & 12.6 & $-19.82 \pm 0.09$ & $0.45 \pm 0.07$ & $0.48 \pm 0.10$ \\
\hline $1304+2818$ & $15.75 \pm 0.01$ & $15.91 \pm 0.04$ & 4.5 & 5.9 & $-20.13 \pm 0.04$ & $0.81 \pm 0.10$ & $0.81 \pm 0.11$ \\
\hline $1306+2938$ & $15.27 \pm 0.02$ & $15.47 \pm 0.04$ & 2.7 & 4.4 & $-20.27 \pm 0.05$ & $0.41 \pm 0.07$ & $0.49 \pm 0.08$ \\
\hline $1306+3111$ & $16.25 \pm 0.04$ & $16.40 \pm 0.09$ & 1.7 & 3.5 & $-18.79 \pm 0.06$ & $0.97 \pm 0.12$ & $0.91 \pm 0.14$ \\
\hline $1307+2910$ & $14.04 \pm 0.03$ & $14.41 \pm 0.05$ & 9.2 & 17.5 & $-21.21 \pm 0.06$ & $1.07 \pm 0.10$ & $0.98 \pm 0.11$ \\
\hline $1308+2958$ & $15.25 \pm 0.01$ & $15.46 \pm 0.03$ & 6.8 & 10.3 & $-20.42 \pm 0.04$ & $0.88 \pm 0.03$ & $0.82 \pm 0.05$ \\
\hline $1308+2950$ & $14.83 \pm 0.06$ & $15.10 \pm 0.06$ & 10.2 & 13.8 & $-21.05 \pm 0.07$ & $1.17 \pm 0.05$ & $1.06 \pm 0.07$ \\
\hline $1310+3027$ & $16.51 \pm 0.04$ & $16.77 \pm 0.06$ & 2.6 & 3.7 & $-19.28 \pm 0.06$ & $0.90 \pm 0.09$ & $0.89 \pm 0.11$ \\
\hline $1312+3040$ & $15.49 \pm 0.04$ & $15.67 \pm 0.07$ & 2.8 & 4.6 & $-20.05 \pm 0.06$ & $0.96 \pm 0.11$ & $0.90 \pm 0.12$ \\
\hline $1312+2954$ & $16.10 \pm 0.03$ & $16.24 \pm 0.06$ & 4.4 & 5.6 & $-19.64 \pm 0.05$ & $0.90 \pm 0.05$ & $0.88 \pm 0.07$ \\
\hline $1313+2938$ & $16.68 \pm 0.05$ & $16.82 \pm 0.13$ & 1.6 & 1.7 & $-20.18 \pm 0.06$ & $0.06 \pm 0.10$ & $0.39 \pm 0.16$ \\
\hline $1314+2827$ & $16.14 \pm 0.03$ & $16.35 \pm 0.06$ & 1.9 & 2.8 & $-19.83 \pm 0.05$ & $0.07 \pm 0.09$ & $0.60 \pm 0.11$ \\
\hline $1320+2727$ & $17.41 \pm 0.07$ & $17.53 \pm 0.09$ & 1.3 & 1.9 & $-18.52 \pm 0.08$ & $-0.03 \pm 0.05$ & $0.39 \pm 0.10$ \\
\hline $1324+2926$ & $17.62 \pm 0.08$ & $18.02 \pm 0.10$ & 0.8 & 2.1 & $-17.46 \pm 0.10$ & $0.63 \pm 0.11$ & $0.89 \pm 0.14$ \\
\hline $1324+2651$ & $15.10 \pm 0.06$ & $15.20 \pm 0.10$ & 1.9 & 2.9 & $-20.79 \pm 0.07$ & $0.44 \pm 0.05$ & $0.61 \pm 0.11$ \\
\hline $1331+2900$ & $18.81 \pm 0.12$ & $19.12 \pm 0.17$ & 1.4 & 1.4 & $-17.87 \pm 0.13$ & $0.39 \pm 0.11$ & $0.50 \pm 0.19$ \\
\hline $1428+2727$ & $14.78 \pm 0.02$ & $14.91 \pm 0.03$ & 2.3 & 5.3 & $-19.98 \pm 0.06$ & $0.50 \pm 0.11$ & $0.44 \pm 0.12$ \\
\hline $1429+2645$ & $17.31 \pm 0.09$ & $17.83 \pm 0.09$ & 2.4 & 3.3 & $-19.17 \pm 0.10$ & $0.43 \pm 0.08$ & $0.64 \pm 0.10$ \\
\hline $1430+2947$ & $16.46 \pm 0.06$ & $16.91 \pm 0.08$ & 3.2 & 4.2 & $-19.76 \pm 0.07$ & $0.90 \pm 0.09$ & $0.79 \pm 0.11$ \\
\hline $1431+2854$ & $15.51 \pm 0.06$ & $15.64 \pm 0.08$ & 5.3 & 5.0 & $-20.85 \pm 0.07$ & $0.96 \pm 0.13$ & $0.81 \pm 0.14$ \\
\hline $1431+2702$ & $16.57 \pm 0.07$ & $17.12 \pm 0.08$ & 2.6 & 3.5 & $-20.28 \pm 0.07$ & $0.50 \pm 0.07$ & $0.55 \pm 0.09$ \\
\hline $1431+2947$ & $17.49 \pm 0.07$ & $18.23 \pm 0.09$ & 2.4 & 5.2 & $-18.12 \pm 0.09$ & $0.59 \pm 0.10$ & $0.55 \pm 0.12$ \\
\hline $1431+2814$ & $16.92 \pm 0.03$ & $17.05 \pm 0.05$ & 2.6 & 2.6 & $-19.51 \pm 0.05$ & $1.01 \pm 0.05$ & $1.04 \pm 0.07$ \\
\hline $1432+2645$ & $15.35 \pm 0.02$ & $15.66 \pm 0.05$ & 7.4 & 9.3 & $-21.04 \pm 0.04$ & $0.72 \pm 0.07$ & $0.74 \pm 0.07$ \\
\hline $1440+2521 \mathrm{~S}$ & $16.80 \pm 0.04$ & $17.12 \pm 0.04$ & 3.3 & 3.7 & $-19.67 \pm 0.05$ & $0.74 \pm 0.05$ & $0.73 \pm 0.06$ \\
\hline $1440+2511$ & $16.37 \pm 0.06$ & $17.07 \pm 0.08$ & 6.4 & 8.7 & $-20.22 \pm 0.07$ & $0.95 \pm 0.07$ & $0.88 \pm 0.09$ \\
\hline $1440+2521 \mathrm{~N}$ & $16.64 \pm 0.03$ & $16.84 \pm 0.03$ & 3.6 & 4.1 & $-19.83 \pm 0.05$ & $1.11 \pm 0.05$ & $0.97 \pm 0.06$ \\
\hline $1442+2845$ & $15.29 \pm 0.02$ & $15.49 \pm 0.02$ & 1.9 & 6.0 & $-18.81 \pm 0.07$ & $0.63 \pm 0.02$ & $0.66 \pm 0.04$ \\
\hline $1443+2714$ & $15.49 \pm 0.10$ & $15.80 \pm 0.14$ & 3.8 & 5.4 & $-20.79 \pm 0.11$ & $0.94 \pm 0.15$ & $0.78 \pm 0.15$ \\
\hline $1443+2844$ & $15.65 \pm 0.02$ & $15.73 \pm 0.04$ & 4.0 & 4.9 & $-20.51 \pm 0.05$ & $0.82 \pm 0.08$ & $0.74 \pm 0.08$ \\
\hline $1443+2548$ & $15.75 \pm 0.03$ & $15.80 \pm 0.06$ & 4.8 & 4.8 & $-20.99 \pm 0.05$ & $0.63 \pm 0.04$ & $0.57 \pm 0.07$ \\
\hline $1444+2923$ & $16.39 \pm 0.03$ & $17.13 \pm 0.04$ & 4.9 & 6.3 & $-19.76 \pm 0.05$ & $0.74 \pm 0.09$ & $0.69 \pm 0.10$ \\
\hline $1452+2754$ & $16.32 \pm 0.04$ & $16.46 \pm 0.04$ & 3.8 & 4.1 & $-20.32 \pm 0.06$ & $0.99 \pm 0.10$ & $0.89 \pm 0.11$ \\
\hline $1506+1922$ & $15.93 \pm 0.03$ & $16.23 \pm 0.02$ & 2.8 & 5.6 & $-19.60 \pm 0.05$ & $1.01 \pm 0.03$ & $1.00 \pm 0.04$ \\
\hline $1513+2012$ & $15.79 \pm 0.09$ & $15.96 \pm 0.13$ & 4.0 & 3.4 & $-21.09 \pm 0.10$ & $0.84 \pm 0.15$ & $0.79 \pm 0.15$ \\
\hline $1537+2506 \mathrm{~N}$ & $15.13 \pm 0.03$ & $15.33 \pm 0.03$ & 4.8 & 6.6 & $-20.76 \pm 0.05$ & $0.90 \pm 0.08$ & $0.87 \pm 0.09$ \\
\hline $1537+2506 \mathrm{~S}$ & $16.10 \pm 0.05$ & $16.32 \pm 0.05$ & 2.9 & 3.5 & $-19.79 \pm 0.07$ & $0.75 \pm 0.09$ & $0.67 \pm 0.10$ \\
\hline $1557+1423$ & $16.65 \pm 0.09$ & $16.83 \pm 0.12$ & 2.6 & 3.3 & $-19.55 \pm 0.10$ & $0.90 \pm 0.14$ & $0.87 \pm 0.15$ \\
\hline $1612+1308$ & $18.05 \pm 0.05$ & $18.11 \pm 0.08$ & 0.5 & 1.4 & $-16.27 \pm 0.08$ & $0.35 \pm 0.12$ & $0.37 \pm 0.15$ \\
\hline $1646+2725$ & $18.16 \pm 0.03$ & $18.54 \pm 0.05$ & 2.8 & 4.5 & $-18.61 \pm 0.05$ & $0.33 \pm 0.20$ & $0.27 \pm 0.21$ \\
\hline $1647+2950$ & $15.43 \pm 0.03$ & $15.56 \pm 0.05$ & 4.7 & 5.7 & $-20.91 \pm 0.05$ & $0.67 \pm 0.11$ & $0.66 \pm 0.12$ \\
\hline $1647+2729$ & $16.03 \pm 0.06$ & $16.06 \pm 0.09$ & 4.1 & 3.9 & $-20.91 \pm 0.07$ & $0.73 \pm 0.10$ & $0.68 \pm 0.12$ \\
\hline $1647+2727$ & $17.12 \pm 0.04$ & $16.15 \pm 0.06$ & 4.3 & 2.0 & $-19.83 \pm 0.06$ & $0.83 \pm 0.09$ & $0.74 \pm 0.10$ \\
\hline $1648+2855$ & $15.40 \pm 0.02$ & $15.58 \pm 0.04$ & 3.2 & 3.8 & $-21.13 \pm 0.04$ & $0.42 \pm 0.07$ & $0.48 \pm 0.08$ \\
\hline $1653+2644$ & $14.72 \pm 0.03$ & $15.01 \pm 0.06$ & 3.8 & 4.5 & $-22.37 \pm 0.05$ & & - \\
\hline $1654+2812$ & $18.06 \pm 0.11$ & $18.60 \pm 0.15$ & 3.6 & 3.5 & $-18.84 \pm 0.12$ & $0.63 \pm 0.08$ & $0.63 \pm 0.11$ \\
\hline
\end{tabular}


Table 3. continued

\begin{tabular}{|c|c|c|c|c|c|c|c|}
\hline $\begin{array}{l}\text { UCM name } \\
\text { (1) }\end{array}$ & $\begin{array}{c}\left(m_{B}\right)_{\mathrm{T}} \\
(2) \\
\end{array}$ & $\begin{array}{c}\left(m_{B}\right)_{24} \\
(3) \\
\end{array}$ & $\begin{array}{c}R_{\mathrm{e}}(\mathrm{kpc}) \\
(4)\end{array}$ & $\begin{array}{c}r_{1 / 2}\left({ }^{\prime \prime}\right) \\
(5)\end{array}$ & $\begin{array}{c}M_{B} \\
(6) \\
\end{array}$ & $\begin{array}{c}(B-r)_{\text {ef }} \\
(7)\end{array}$ & $\begin{array}{c}(B-r)_{24} \\
(8)\end{array}$ \\
\hline $1655+2755$ & $\overline{c 15.59 \pm 0.09}$ & $\overline{15.88 \pm 0.13}$ & $\overline{9.9}$ & $\overline{10.0}$ & $-21.30 \pm 0.10$ & $\overline{c 1.28 \pm 0.15}$ & $1.20 \pm 0.15$ \\
\hline $1656+2744$ & $16.84 \pm 0.03$ & $17.37 \pm 0.23$ & 1.7 & 3.0 & $-19.89 \pm 0.04$ & $0.74 \pm 0.13$ & $0.82 \pm 0.26$ \\
\hline $1657+2901$ & $17.12 \pm 0.01$ & $17.23 \pm 0.04$ & 2.2 & 2.3 & $-19.54 \pm 0.04$ & $0.73 \pm 0.10$ & $0.67 \pm 0.11$ \\
\hline $1659+2928$ & $15.73 \pm 0.09$ & $16.02 \pm 0.12$ & 4.9 & 4.4 & $-21.24 \pm 0.10$ & $0.76 \pm 0.14$ & $0.76 \pm 0.14$ \\
\hline $1701+3131$ & $15.27 \pm 0.08$ & $15.40 \pm 0.11$ & 4.2 & 4.2 & $-21.47 \pm 0.08$ & $0.99 \pm 0.12$ & $0.82 \pm 0.13$ \\
\hline $2238+2308$ & $14.86 \pm 0.02$ & $14.86 \pm 0.05$ & 5.4 & 5.6 & $-21.17 \pm 0.05$ & $0.81 \pm 0.07$ & $0.77 \pm 0.08$ \\
\hline $2239+1959$ & $14.82 \pm 0.04$ & $15.01 \pm 0.05$ & 3.0 & 4.4 & $-21.34 \pm 0.05$ & $0.78 \pm 0.09$ & $0.73 \pm 0.10$ \\
\hline $2249+2149$ & $15.96 \pm 0.06$ & $16.26 \pm 0.06$ & 7.9 & 5.9 & $-21.51 \pm 0.07$ & $1.27 \pm 0.34$ & $1.20 \pm 0.35$ \\
\hline $2250+2427$ & $15.39 \pm 0.03$ & $15.48 \pm 0.06$ & 3.0 & 2.6 & $-21.88 \pm 0.04$ & $0.32 \pm 0.08$ & $0.55 \pm 0.10$ \\
\hline $2251+2352$ & $16.36 \pm 0.02$ & $16.45 \pm 0.05$ & 1.8 & 2.4 & $-19.81 \pm 0.04$ & $0.50 \pm 0.10$ & $0.59 \pm 0.11$ \\
\hline $2253+2219$ & $16.12 \pm 0.02$ & $16.18 \pm 0.08$ & 1.9 & 3.7 & $-19.88 \pm 0.05$ & $0.61 \pm 0.05$ & $0.63 \pm 0.09$ \\
\hline $2255+1930 \mathrm{~S}$ & $16.08 \pm 0.02$ & $16.16 \pm 0.07$ & 1.2 & 2.1 & $-19.54 \pm 0.05$ & $0.21 \pm 0.21$ & $0.56 \pm 0.22$ \\
\hline $2255+1930 \mathrm{~N}$ & $15.76 \pm 0.02$ & $15.95 \pm 0.05$ & 2.2 & 3.8 & $-19.80 \pm 0.05$ & $1.02 \pm 0.20$ & $1.10 \pm 0.21$ \\
\hline $2255+1926$ & $16.74 \pm 0.03$ & $17.18 \pm 0.06$ & 3.2 & 5.5 & $-18.77 \pm 0.06$ & $0.55 \pm 0.10$ & $0.54 \pm 0.11$ \\
\hline $2255+1654$ & $16.62 \pm 0.03$ & $16.84 \pm 0.06$ & 6.8 & 10.2 & $-20.50 \pm 0.04$ & $1.20 \pm 0.05$ & $1.08 \pm 0.08$ \\
\hline $2256+2001$ & $15.62 \pm 0.04$ & $16.03 \pm 0.04$ & 8.2 & 12.2 & $-20.35 \pm 0.06$ & $1.11 \pm 0.07$ & $1.06 \pm 0.08$ \\
\hline $2257+2438$ & $16.04 \pm 0.05$ & $16.15 \pm 0.10$ & 1.3 & 1.4 & $-20.71 \pm 0.06$ & $-0.30 \pm 0.10$ & $0.14 \pm 0.12$ \\
\hline $2257+1606$ & $16.40 \pm 0.07$ & $16.57 \pm 0.15$ & 2.0 & 2.1 & $-20.38 \pm 0.08$ & - & - \\
\hline $2258+1920$ & $15.80 \pm 0.05$ & $15.99 \pm 0.09$ & 2.8 & 4.6 & $-19.95 \pm 0.06$ & $0.33 \pm 0.10$ & $0.41 \pm 0.11$ \\
\hline $2300+2015$ & $16.53 \pm 0.04$ & $16.87 \pm 0.06$ & 4.4 & 3.9 & $-20.27 \pm 0.05$ & $0.70 \pm 0.11$ & $0.80 \pm 0.12$ \\
\hline $2302+2053 \mathrm{~W}$ & $17.97 \pm 0.02$ & $18.20 \pm 0.08$ & 1.6 & 1.7 & $-18.79 \pm 0.04$ & $0.53 \pm 0.06$ & $0.86 \pm 0.10$ \\
\hline $2302+2053 \mathrm{E}$ & $15.49 \pm 0.02$ & $15.85 \pm 0.06$ & 5.6 & 6.8 & $-21.28 \pm 0.04$ & $1.49 \pm 0.05$ & $1.37 \pm 0.07$ \\
\hline $2303+1856$ & $15.62 \pm 0.03$ & $15.86 \pm 0.17$ & 3.5 & 5.1 & $-20.64 \pm 0.05$ & $0.90 \pm 0.11$ & $0.85 \pm 0.20$ \\
\hline $2303+1702$ & $17.46 \pm 0.05$ & $17.76 \pm 0.11$ & 4.3 & 4.3 & $-19.86 \pm 0.06$ & $1.31 \pm 0.12$ & $1.27 \pm 0.14$ \\
\hline $2304+1640$ & $17.56 \pm 0.02$ & $17.89 \pm 0.17$ & 0.9 & 2.1 & $-17.81 \pm 0.05$ & $-0.18 \pm 0.10$ & $0.27 \pm 0.19$ \\
\hline $2304+1621$ & $16.69 \pm 0.08$ & $17.28 \pm 0.14$ & 2.9 & 4.0 & $-20.34 \pm 0.08$ & $1.22 \pm 0.12^{\dagger}$ & - \\
\hline $2307+1947$ & $16.62 \pm 0.05$ & $16.85 \pm 0.06$ & 2.9 & 3.7 & $-19.62 \pm 0.06$ & $1.01 \pm 0.21$ & $0.91 \pm 0.21$ \\
\hline $2310+1800$ & $16.76 \pm 0.03$ & $16.97 \pm 0.05$ & 3.7 & 3.6 & $-20.18 \pm 0.05$ & $0.78 \pm 0.33$ & $0.94 \pm 0.33$ \\
\hline $2312+2204$ & $17.13 \pm 0.08$ & $17.39 \pm 0.10$ & 2.1 & 2.6 & $-19.64 \pm 0.08$ & 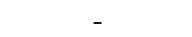 & . \\
\hline $2313+1841$ & $16.59 \pm 0.06$ & $17.27 \pm 0.12$ & 3.1 & 8.8 & $-19.87 \pm 0.07$ & $0.87 \pm 0.06$ & $0.79 \pm 0.13$ \\
\hline $2313+2517$ & $14.91 \pm 0.03$ & $15.23 \pm 0.04$ & 5.7 & 6.4 & $-21.45 \pm 0.05$ & 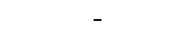 & 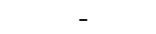 \\
\hline $2315+1923$ & $17.22 \pm 0.07$ & $17.61 \pm 0.16$ & 2.2 & 2.6 & $-19.73 \pm 0.08$ & $0.39 \pm 0.09$ & $0.48 \pm 0.18$ \\
\hline $2316+2457$ & $14.35 \pm 0.03$ & $14.51 \pm 0.05$ & 4.7 & 7.0 & $-22.03 \pm 0.05$ & $0.86 \pm 0.09$ & $0.77 \pm 0.09$ \\
\hline $2316+2459$ & $15.82 \pm 0.04$ & $16.24 \pm 0.11$ & 6.4 & 10.6 & $-20.53 \pm 0.05$ & $0.85 \pm 0.08$ & $0.83 \pm 0.13$ \\
\hline $2316+2028$ & $16.84 \pm 0.04$ & $17.08 \pm 0.17$ & 1.3 & 1.9 & $-19.33 \pm 0.06$ & $-0.38 \pm 0.09$ & $0.12 \pm 0.20$ \\
\hline $2317+2356$ & $13.86 \pm 0.04$ & $13.99 \pm 0.11$ & 8.8 & 9.8 & $-22.89 \pm 0.05$ & $0.75 \pm 0.05$ & $0.67 \pm 0.12$ \\
\hline $2319+2234$ & $16.50 \pm 0.05$ & $16.86 \pm 0.13$ & 3.2 & 3.2 & $-20.36 \pm 0.06$ & $-0.17 \pm 0.08$ & $0.12 \pm 0.15$ \\
\hline $2319+2243$ & $15.69 \pm 0.05$ & $16.05 \pm 0.12$ & 4.4 & 5.4 & $-20.83 \pm 0.06$ & $1.07 \pm 0.07$ & $1.02 \pm 0.14$ \\
\hline $2320+2428$ & $15.13 \pm 0.06$ & $15.80 \pm 0.16$ & 6.6 & 10.4 & $-21.51 \pm 0.07$ & $1.26 \pm 0.07$ & $1.21 \pm 0.17$ \\
\hline $2321+2149$ & $16.55 \pm 0.05$ & $16.74 \pm 0.09$ & 4.2 & 5.0 & $-20.36 \pm 0.06$ & $0.66 \pm 0.10$ & $0.66 \pm 0.11$ \\
\hline $2321+2506$ & $15.75 \pm 0.05$ & $15.86 \pm 0.09$ & 5.3 & 5.3 & $-20.86 \pm 0.06$ & $0.54 \pm 0.10$ & $0.51 \pm 0.11$ \\
\hline $2322+2218$ & $17.69 \pm 0.02$ & $17.89 \pm 0.03$ & 1.9 & 2.4 & $-18.32 \pm 0.05$ & $0.77 \pm 0.33$ & $0.95 \pm 0.33$ \\
\hline $2324+2448$ & $13.32 \pm 0.07$ & $13.62 \pm 0.08$ & 7.1 & 21.4 & $-21.20 \pm 0.10$ & $0.91 \pm 0.10$ & $0.81 \pm 0.11$ \\
\hline $2325+2318$ & $13.21 \pm 0.01$ & $13.37 \pm 0.03$ & 3.1 & 9.9 & $-21.25 \pm 0.06$ & & \\
\hline $2325+2208$ & $12.91 \pm 0.04$ & $13.00 \pm 0.08$ & 9.9 & 28.8 & $-21.67 \pm 0.07$ & $1.05 \pm 0.11$ & $0.98 \pm 0.10$ \\
\hline $2326+2435$ & $16.09 \pm 0.04$ & $16.54 \pm 0.14$ & 2.7 & 9.3 & $-19.17 \pm 0.07$ & $0.48 \pm 0.08$ & $0.44 \pm 0.16$ \\
\hline $2327+2515 \mathrm{~N}$ & $15.83 \pm 0.05$ & $15.47 \pm 0.06$ & 2.9 & 2.3 & $-19.79 \pm 0.07$ & $0.34 \pm 0.07$ & $0.10 \pm 0.09$ \\
\hline $2327+2515 \mathrm{~S}$ & $15.77 \pm 0.05$ & $15.64 \pm 0.06$ & 2.7 & 3.3 & $-19.85 \pm 0.07$ & $0.60 \pm 0.07$ & $0.46 \pm 0.09$ \\
\hline $2329+2427$ & $16.03 \pm 0.08$ & $16.39 \pm 0.09$ & 3.5 & 5.9 & $-19.52 \pm 0.09$ & $1.50 \pm 0.10$ & $1.45 \pm 0.11$ \\
\hline $2329+2500$ & $16.28 \pm 0.04$ & $16.47 \pm 0.09$ & 1.9 & 2.7 & $-20.16 \pm 0.06$ & $0.89 \pm 0.12$ & $0.99 \pm 0.14$ \\
\hline $2329+2512$ & $17.00 \pm 0.07$ & $17.43 \pm 0.08$ & 1.0 & 2.9 & $-17.63 \pm 0.09$ & $1.16 \pm 0.32$ & $1.15 \pm 0.33$ \\
\hline $2331+2214$ & $17.63 \pm 0.07$ & $17.99 \pm 0.10$ & 2.9 & 3.4 & $-19.11 \pm 0.08$ & $1.36 \pm 0.08$ & $1.34 \pm 0.11$ \\
\hline $2333+2248$ & $17.45 \pm 0.06$ & $17.79 \pm 0.13$ & 4.6 & 3.9 & $-19.60 \pm 0.07$ & $1.19 \pm 0.16$ & $1.09 \pm 0.15$ \\
\hline $2333+2359$ & $17.70 \pm 0.06$ & $18.01 \pm 0.10$ & 3.0 & 2.8 & $-19.08 \pm 0.07$ & $1.98 \pm 0.13$ & $1.91 \pm 0.14$ \\
\hline $2348+2407$ & $17.93 \pm 0.08$ & $18.25 \pm 0.10$ & 2.0 & 2.1 & $-18.88 \pm 0.09$ & $1.63 \pm 0.10$ & $1.64 \pm 0.13$ \\
\hline $2351+2321$ & $18.56 \pm 0.08$ & $18.80 \pm 0.10$ & 1.1 & 1.5 & $-17.69 \pm 0.09$ & $1.81 \pm 0.17$ & $2.06 \pm 0.18$ \\
\hline
\end{tabular}

$\dagger$ Total colour calculated from integrated magnitudes. No radial data are available due to low SNR in the images.

Table 3. (1) UCM name. (2) Total Johnson $B$ magnitude calculated with several polygons. We have also measured the asymptotic magnitude at two Kron radius yielding an average difference with the polygon measure of 0.02 magnitudes. (3) Johnson $B$ magnitude measured inside the $24 \mathrm{mag} \operatorname{arcsec}^{-2}$ isophote. (4) Effective radius in kpc measured in circular apertures and converted to distance using a Hubble constant $H_{0}=50 \mathrm{~km} \mathrm{~s}^{-1} \mathrm{Mpc}^{-1}$ and a deceleration parameter $q_{0}=0.5$. (5) Equivalent half-light radius calculated with isophotal apertures. (6) Absolute magnitude corrected from Galactic extinction according to the maps of Galactic reddening of Burstein \& Heiles (1982). (7) $B-r$ colour measured inside the effective isophote. (8) $B-r$ colour measured inside the $24 \mathrm{mag} \operatorname{arcsec}^{-2}$ isophote. 


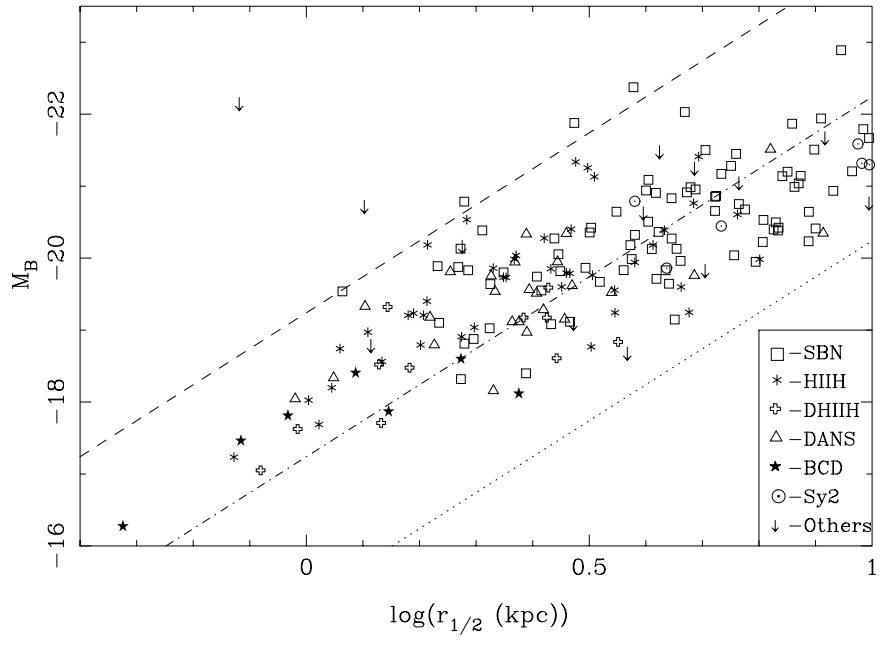

Fig. 2. Absolute magnitude corrected from Galactic extinction versus effective radius, measured in $\mathrm{kpc}$ as the circular aperture that contains half the light of the galaxy. As reference, constant surface brightness lines corresponding to $-14,-16$ and $-18 \mathrm{mag} \mathrm{kpc}^{-2}$ are plotted

types, whereas the HII-like includes HIIH, DHIIH and BCD type galaxies.

The UCM Survey does not contain objects brighter than an absolute magnitude of -22.9 or fainter than -16.3 . Despite the considerable scatter, we observe a correlation between $M_{B}, r_{1 / 2}$ and the spectroscopic type in Fig. 2. BCD galaxies appear as small and faint objects in the bottom left corner of the plot. SBN galaxies are more concentrated in the largest effective radius and luminosity zone of the diagram. This should be the place for normal grand-design spirals. The existence of a bright starburst in the nucleus of SBN objects turns them into objects redder than those with the starburst located out of the nucleus (see below the discussion of Figs. 4 and 6). Only UCM 1612+1308 shows the typical small size of nucleated compact dwarfs. Most of the DANS and HIIH galaxies are also located in the small effective radii zone, below $5 \mathrm{kpc}$.

As reference, we have plotted the constant surface brightness lines corresponding to $-14,-16$ and $-18 \mathrm{mag} \mathrm{kpc}^{-2}$ in Fig. 2 .

In Fig. 3 we plot the histograms of $(B-r)_{\text {ef }}$ colours of UCM galaxies corrected from Galactic extinction according to their morphological (Vitores et al. 1996a) and spectroscopic classification (Gallego et al. 1996). The averaged colours of each Hubble type are listed in Table 4, jointly with the mean colours calculated by Fukugita et al. (1995). The vertical ticks in these diagrams show Fukugita et al. (1995) colours and averaged colours for each spectroscopic type.

Overall, early-type spirals show a bluer colour than those of Fukugita et al. (1995), probably related to the presence of the star-forming process. On the other hand, irregulars and BCDs do show redder $B-r$ colours than Fukugita's sample; this could be a selection effect, given
Table 4. Mean colours according to Hubble type

\begin{tabular}{cccc}
\hline $\begin{array}{c}\text { Hubble type } \\
(1)\end{array}$ & $\begin{array}{c}\overline{(B-r)}_{\mathrm{UCM}} \\
(2)\end{array}$ & $\begin{array}{c}\overline{(B-r)}_{\mathrm{F} 95} \\
(3)\end{array}$ & $\begin{array}{c}N_{\text {gal }} \\
(4)\end{array}$ \\
\hline \hline $\mathrm{Sa}$ & 0.74 & $0.97(\mathrm{Sab})$ & 40 \\
$\mathrm{Sb}$ & 0.75 & $0.73(\mathrm{Sbc})$ & 44 \\
$\mathrm{Sc}+$ & 0.72 & $0.65(\mathrm{Scd})$ & 45 \\
$\mathrm{Irr}$ & 0.42 & $0.24(\mathrm{Irr})$ & 8 \\
$\mathrm{BCD}$ & 0.34 & $0.24(\mathrm{Irr})$ & 4 \\
\hline \hline
\end{tabular}

Table 4. (1) Hubble type. (2) Mean total $B-r$ colours of the UCM sample. (3) Mean total $B-r$ colours tabulated in Fukugita et al. (1995). (4) Number of galaxies used in the calculated mean colours.

that very blue objects would not show up at the original objective-prism plates as they were taken in the $\mathrm{H} \alpha$ region.

Although the spectroscopic histograms show a great dispersion we observe that SBN galaxies are redder than other types. The bluest objects appear to be BCDs and DHIIHs. These two facts could be explained in two different ways: SBNs could be affected by larger dust reddening or the starburst could be more relevant in BCD and DHIIH galaxies, making them bluer. In fact, Gallego et al. (1997) showed that the mean $B-V$ colour excess for SBN galaxies is $0.2^{\mathrm{m}}$ higher than for HII-like galaxies.

Both kind of data are mixed in Fig. 4. SBN galaxies dominate the spiral zone (from $T=1$-Sa- to $T=6$-Sc-), adding a great colour dispersion to our sample. There are also 7 very blue objects, all of them late-type spirals $(\mathrm{Sc}+)$ or irregulars. some of these objects are low metallicity galaxies, for example UCM $2304+1640((B-r))_{\mathrm{ef}}=-0.18$, metallicity $\left.Z_{\odot} / 7\right)$ or UCM $0049+0017\left((B-r)_{\mathrm{ef}}=-0.33\right.$, metallicity $\left.Z_{\odot} / 20\right)$.

The $B-r$ histogram for the whole sample is plotted in Fig. 5. The averaged effective colour of the UCM sample is $0.73 \pm 0.41$. The distribution is rather flat, being dominated by galaxies with a colour corresponding to a typical spiral.

In Fig. 6 we plot the $B$ absolute magnitude $M_{B}$ versus the effective colour $(B-r)_{\text {ef }}$. Labels correspond to the spectroscopic type of each object. An extinction vector of 0.4 magnitudes in the $B$ band has been drawn. SBN galaxies are located in the most luminous and reddest part of the plot, jointly with Sy2 galaxies. In the other hand, BCDs appear to be the bluest and faintest objects in our sample. UCM objects are compared with a normal sample of galaxies from the literature in Fig. 7; we have selected common galaxies in the Nearby Universe from the NGC, IC and Mrk catalogs extracted from the NED database ${ }^{2}$.

${ }^{2}$ The NASA/IPAC Extragalactic Database (NED) is operated by the Jet Propulsion Laboratory, California Institute of Technology, under contract with the National Aeronautics and Space Administration. 

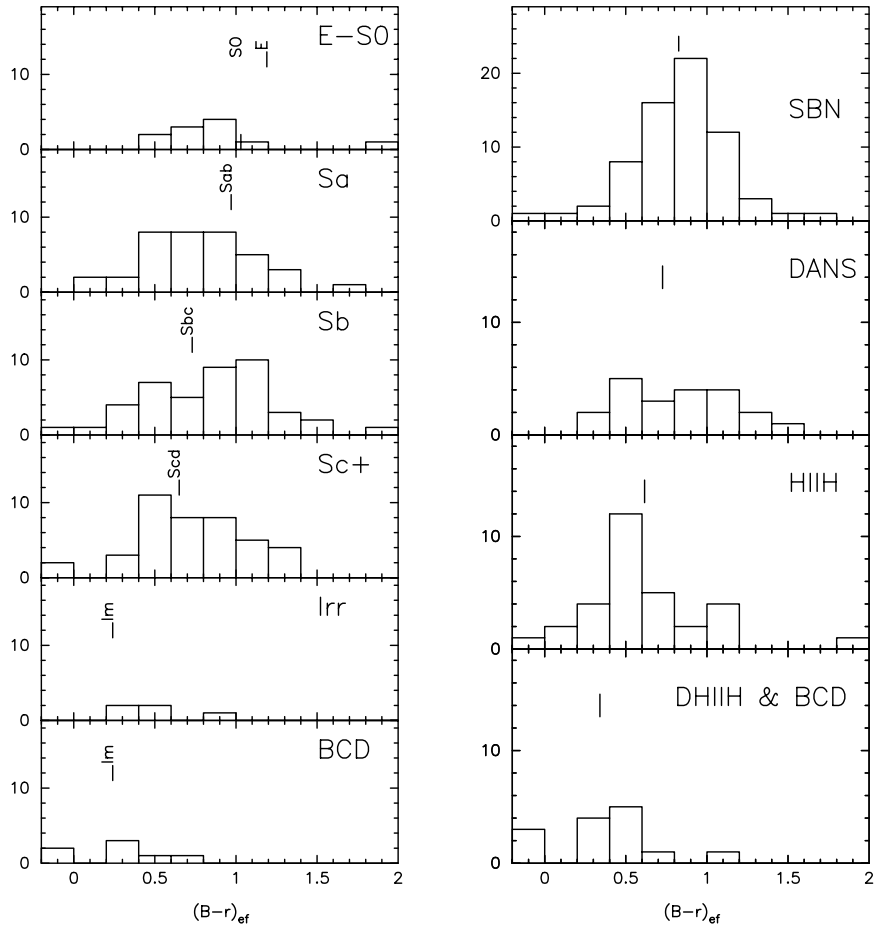

Fig. 3. Histograms of the $(B-r)_{\text {ef }}$ colours of the UCM galaxies corrected from Galactic extinction according to their morphological and spectroscopic classification as established in Vitores et al. (1996a) and Gallego et al. (1996), respectively. The vertical marks in the left diagram are the typical colours of each morphological type as tabulated in Fukugita et al. (1995); mean colours are listed in Table 4. In the right diagram we have marked the averaged colour of each spectroscopic type. The values are: 0.83 for SBN type, 0.73 for DANS, 0.62 for HIIH, 0.34 for DHIIH \& BCD

The BCD data have been extracted from Doublier et al. (1997). Both sets of reference data are drawn lightened.

In the top panel we have compared our colours with those of spirals. As expected, most of the UCM sample is located in the region where normal spiral galaxies are found in this colour-magnitude diagram; some of our galaxies have similar colours to those of early-type galaxies though this could be due to internal reddening. The BCD galaxies in our sample seem to be about $0.7^{\mathrm{m}}$ brighter and $0.2^{\mathrm{m}}$ bluer than the Doublier et al. (1997) sample.

\section{Summary}

We have presented optical photometry in the Johnson $B$ and Gunn $r$ bands of the UCM Survey, a local sample of star-forming galaxies. The optical colours of UCM galaxies have been compared with the literature. Though there is a great dispersion in our data, statistically there is a good correlation between multiband photometric, morphological and spectroscopic properties.

Optical colours for the UCM galaxies, when compared with those calculated by Fukugita et al. (1995) according

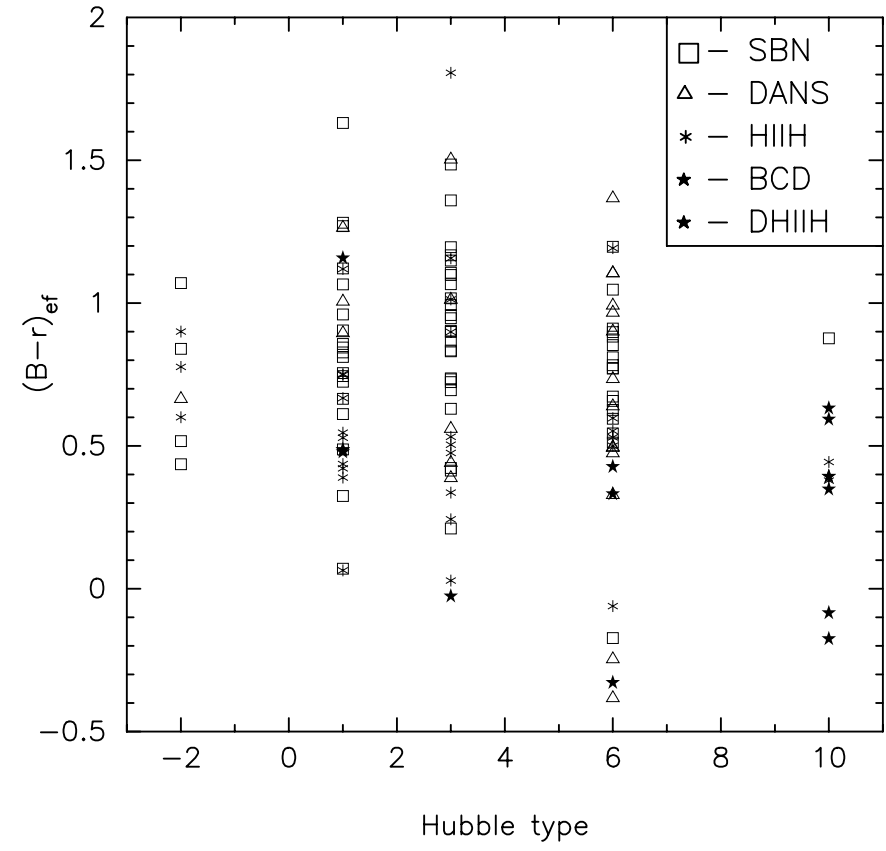

Fig. 4. Relation between spectroscopic and morphological types and $(B-r)_{\text {ef }}$ colour. We have selected the main spectroscopic types of our sample: SBN, DANS, HIIH and BCD \& DHIIH, as classified in Gallego et al. (1996) and morphological types from S0 to Irr; the galaxies classified as Sc+ by Vitores et al. (1996a) are included in $T=6$-corresponding to a Sc galaxy

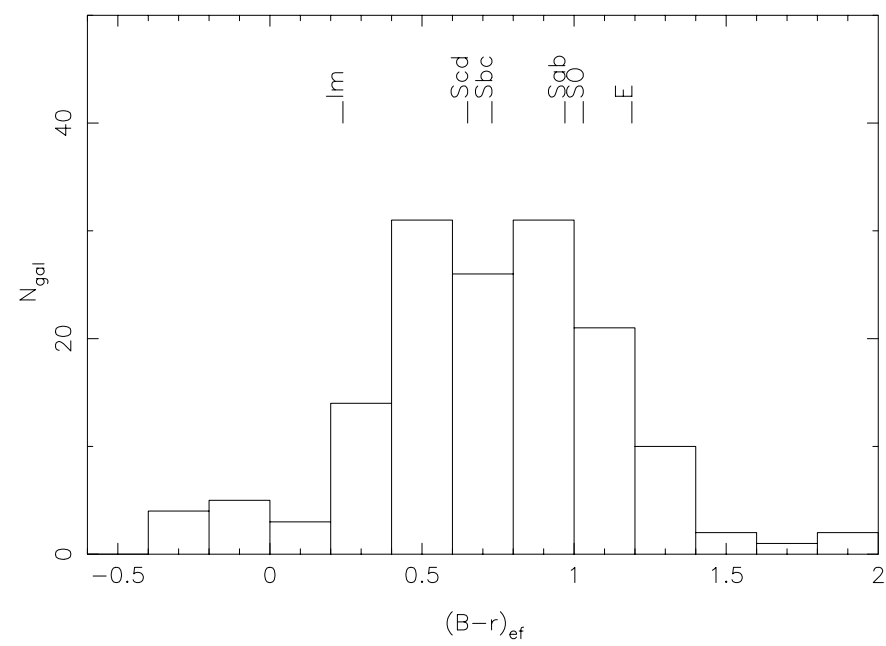

Fig. 5. $B-r$ histogram of the UCM Survey Lists I and II. The averaged colours of Fukugita et al. (1995) have been marked at the top

to their Hubble type, seem to be slightly bluer in earlytype spirals and redder in irregulars and BCD's.

Related to the spectroscopic properties of our galaxies, the calculated colours show the reddening of the objects whose $\mathrm{H} \alpha$ emission is associated with the nucleus of the galaxy (SBN or Sy). We have also noticed that, as expected, low metallicity objects seem to be the bluest ones.

In next papers we will study the morphological properties of the UCM sample in the Johnson $B$ band. Using 


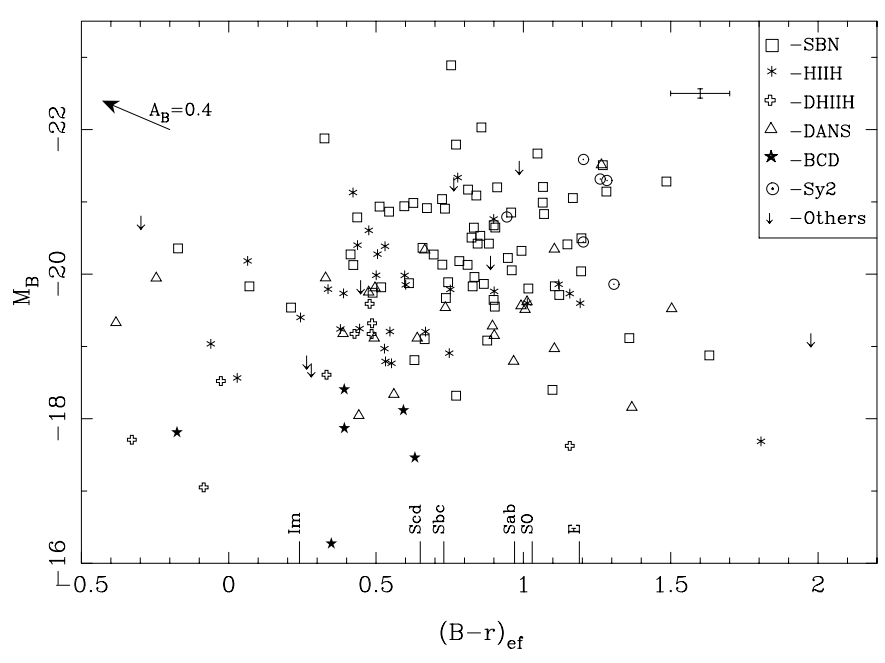

Fig. 6. Absolute $B$ magnitude $M_{B}$ corrected from Galactic extinction versus $(B-r)_{\text {ef }}$ (effective colour). Bottom marks are $B-r$ colours from Fukugita et al. (1995). An extinction vector corresponding to 0.4 magnitudes in the Johnson $B$ band is given and also the averaged error bars of both sets of data

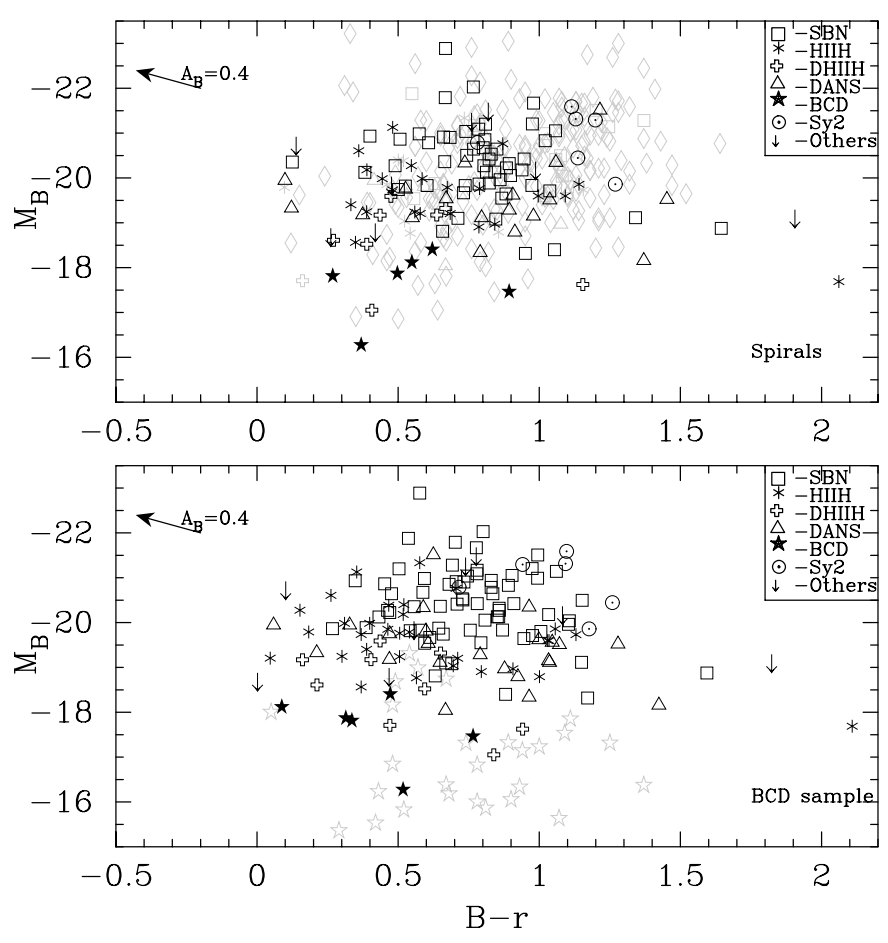

Fig. 7. Colour-magnitude diagram of the UCM survey galaxies compared with other galaxies in the Nearby Universe (drawn lightened as diamonds -spirals- and five-points stars -BCD's-). In the top panel we have used isophote 25 colours while in the second panel we have represented total colours (extracted from the $2 \cdot r_{\text {Kron }}$ aperture). All absolute magnitudes are integrated total magnitudes. Typical error is that shown in Fig. 6 bulge-disk decompositions and $\mathrm{H} \alpha$ images we will perform synthesis models and will compare global properties of our sample with the galaxy population at high redshift.

Acknowledgements. This paper is based on observations obtained at the German-Spanish Astronomical Centre, Calar Alto, Spain, operated by the Max-Planck Institut für Astronomie (MPIA), Heidelberg, jointly with the Spanish Commission for Astronomy. It is also partly based on observations made with the Jacobus Kapteyn Telescope operated on the island of La Palma by the Royal Greenwich Observatory in the Spanish Observatorio del Roque de los Muchachos of the Instituto de Astrofísica de Canarias and the $1.52 \mathrm{~m}$ telescope of the EOCA/OAN Observatory.

This research has made use of the NASA/IPAC Extragalactic Database (NED) which is operated by the Jet Propulsion Laboratory, California Institute of Technology, under contract with the National Aeronautics and Space Administration. We have also use of the LEDA database, http://www-obs.univ-lyon1.fr.

This research was also supported by the Spanish Programa Sectorial de Promoción General del Conocimiento under grants PB96-0610 and PB96-0645.

We would like to thank C.E. García-Dabó and S. Pascual for their help during part of the observing runs.

We are grateful to Dr. M. Fukugita for his helpful remarks that have improved this paper.

\section{References}

Alonso O., García-Dabó E., Zamorano J., Gallego J., Rego M., 1999, ApJS 122, 415

Alonso-Herrero A., Aragón-Salamanca A., Zamorano J., Rego M., 1996, MNRAS 278, 417

Balzano V.A., 1983, ApJ 268, 602

Burstein D., Heiles C., 1982, AJ 87, 1165

Doublier V., Comte G., Petrosian A., Turatto M., Surace C., 1997, A\&AS 124, 405

Fitzpatrick E.L., 1999, PASP 111, 63

Fukugita M., Shimasaku K., Ichikawa T., 1995, PASP 107, 945

Gallego J., Zamorano J., Aragón-Salamanca A., Rego M., 1995, ApJ 455, L1

Gallego J., Zamorano J., Rego M., Alonso O., Vitores A.G., 1996, A\&AS 120, 323

Gallego J., Zamorano J., Rego M., Alonso O., Vitores A.G., 1997, ApJ 475, 502

Gallego J., 1998, in: Thuan T.X., Balkowski C., Cayette V., Tran Tranh Van J. (eds.), Dwarf Galaxies and Cosmologies, Proceedings of the XVIIIth Moriond astrophysics meeting. Éditions Frontières, Gif-sur-Yvette, France

Gallego J., 1999, Ap\&SS 263, 1

Gil de Paz A., Aragón-Salamanca A., Gallego J., AlonsoHerrero A., Zamorano J., Kauffmann G., 1999, MNRAS (accepted)

Guzmán R., Gallego J., Koo D.C., et al., 1997, ApJ 489, 559

Kron R.G., 1980, ApJS 43, 305

Landolt A.U., 1992, AJ 104, 340

Madau P., 1999, Physica Scripta, Proceedings of the Nobel Symposium, Particle Physics and the Universe (Enkoping, Sweden, August, 1998), astro-ph/9902228 
Salzer J.J., MacAlpine G.M., Boroson T.A., 1989, ApJS 70, 479

Stetson P., 1990, PASP 102, 932

Vitores A.G., Zamorano J., Rego M., Alonso O., Gallego J., 1996a, A\&AS 118, 7

Vitores A.G., Zamorano J., Rego M., Gallego J., Alonso O., 1996b, A\&AS 120, 385
Zamorano J., Rego M., Gallego J., Vitores A.G., GonzalezRiestra R., Rodriguez-Caderot G., 1994, ApJS 95, 387

Zamorano J., Gallego J., Rego M., Vitores A.G., Alonso O., 1996, ApJS 105, 343 